\title{
TrackT: Accurate Tracking of RFID Tags with mm-level Accuracy Using First-order Taylor Series Approximation
}

\author{
Zhongqin Wang, Ning Ye, Reza Malekian, Fu Xiao, Ruchuan Wang
}

\begin{abstract}
Radio Frequency Identification (RFID) technology is widely used to achieve indoor object tracking and positioning. Currently, many methods need to deploy a large number of reference tags beforehand and some are limited by antennas' spacing. Further, the signal propagation along Non-Line of Sight introduces multipath effects which will challenge the accuracy of RFID localization system. In this work, we propose a method based on measured phase to track mobile RFID tags with millimeter level (mm-level) accuracy. We first partition the surveillance region into square grids at mm-level and suppose that there is a virtual tag as the same as the tracked one in each grid. On this basis, for the case where the tags move along a known track with constant speed, we only need to locate the tag's initial position. We leverage phase periodicity to obtain some candidates and then eliminate position ambiguity by double difference true phase. And for the case where the tag's moving track is unknown to the system, we adopt a first-order Taylor series expansion to calculate the relative displacements of the tracked tag and then locate the initial position as the same process as tracking the known trajectory. In our experiment, our solution can achieve a mean error distance of $0.26 \mathrm{~cm}$ and $0.55 \mathrm{~cm}$ for known and unknown movement tracks respectively.
\end{abstract}

Index Terms - Phase detection, Radio frequency identification, RFID tags, RF signals.

The research is support by National Natural Science Foundation of P. R. China (No.61572260), Major Program of Jiangsu Higher Education Institutions (No.14KJA520002), the Key Research and Development Program of Jiangsu Province (Social Development Program) (No.BE2015702), Jiangsu Planned Projects for Postdoctoral Research Funds (No.1302055C), China Postdoctoral Science Foundation (No.2014M560440), and National Research Foundation, South Africa (AOX220).

Zhongqin Wang is with the College of Internet of things, Nanjing University of Posts and Telecommunications, Nanjing, 210003, China (e-mail: zhongqin.wang.CN@ieee.org).

Ning Ye is with the College of Computer, Nanjing University of Posts and Telecommunications, Nanjing, 210003, China, and also with the Jiangsu High Technology Research Key Laboratory for Wireless Sensor Networks, Nanjing 210003, China (e-mail: yening @ njupt.edu.cn).

Reza Malekian is with the Department of Electrical, Electronic and Computer Engineering, University of Pretoria, Pretoria, South Africa (e-mail: reza.malekian@up.ac.za).

Fu Xiao and Ruchuan Wang are with the College of Computer, Nanjing University of Posts and Telecommunications, Nanjing, 210003, China, and also with the Jiangsu High Technology Research Key Laboratory for Wireless Sensor Networks, Nanjing 210003, China. He is also with Key Lab of Broadband Wireless Communication and Sensor Network Technology of Ministry of Education, Nanjing University of Posts and Telecommunications, Nanjing, 210003, China (e-mail: \{xiaof, wangrc\} @njupt.edu.cn).

\section{INTRODUCTION}

$\mathrm{R}$ ADIO Frequency Identification (RFID) technology is increasingly used in various applications such as assisted tracking of robots [1], product identification[2], asset assessment, indoor positioning and so on. Currently, the common method to locate passive tags in practice is described as follows. People often deploy many RFID readers in different monitoring areas to continuously read RFID tags. The tags are assigned with the unique Electronic Product Code (EPC) and given position information beforehand in the database. Once a reader captures a new tag's EPC, people consider that the tag has been moved to the reader's surveillance area. However, there are many disadvantages in this coarse method: (i) Low positioning resolution. The reading range of an RFID reader antenna is generally about 3 10 meters, and RFID readers can only provide "absence and presence" results, so the system is far from meeting the high accuracy requirements. (ii) False negative reads [3], [4] and false positive reads [5], [6]. The former means that a reader fails to read a tag in the reading zone and the latter means that a tag in some other areas outside the intended read zone is read. These two problems can also affect the positioning accuracy.

Many applications will benefit from millimeter level (mm-level) localization accuracy. For example, false positive reads will be avoided by setting the intended reading zone beforehand. If the RFID tag is within the area, the RFID reader will record and report events related to this tracked tag. If not, the reader will ignore it. As another example, in a large-scale clothes shop a retailer could use the RFID location system to visually track clothes with RFID tags, making sure that sales representatives could easily find matching clothes which may have gone astray and customers could easily know the locations of wanted clothes.

At present, the two key approaches for RFID localization are based on received signal strength indication (RSSI) and radio frequency (RF) phase.

(i) RSSI. The RSSI methods [7]-[11] need to deploy many reference tags since the absolute calibration for RSSI measurements is rather difficult, and positioning accuracy is greatly affected by antenna design, impedance matching, and the changes in reflection coefficient [12]. The distance error is about $60 \mathrm{~cm}$. At present, many commercial-off-the-shelf (COTS) RFID readers can report RF phase once an RFID tag is 
successfully read. Phase resolution can reach $1.5 \times 10^{-3}$ radians, offering $\left(1.5 \times 10^{-3} \times 32.587\right) / 4 \pi=3.89 \times 10^{-3} \mathrm{~cm}$ ranging resolution for an RF carrier wave at the frequency of 920.625MHz. In addition, the RSSI-based methods are not a good choice because the propagation environment will more easily affect the RSSI measurements than phase [13]. As a result, the method to track tags' movement trajectories based on RF phase with higher resolution and better noise-tolerant ability than RSSI has received many researchers' attention.

(ii) RF Phase. Angle of arrival (AoA) [14]-[16] uses multiple antennas to receive the tag's phase and then computes their angles based on phase difference. But these methods need to put a strict constraint on the antennas' spacing. Backpos [17] deploys more than three antennas to read tags' phase, where two adjacent antennas should be within a spacing of half a wavelength. After that, they use the hyperbolic positioning method to locate tags. However, if adjacent antennas' spacing is greater than half a wavelength, the method will fail to deal with the phase periodicity, leading to numerous possible candidates. PinIt [18] captures and extracts multipath profiles of the target tag and reference tags at known positions via an antenna motion, and then adopts dynamic time warping (DTW) techniques to pinpoint a tag's location. However, it needs to deploy dense reference tags in advance and can't track mobile tags. DAH (Differential Augmented Hologram) [19] can track known and unknown trajectories of mobile tags with a mean error distance of $0.6 \mathrm{~cm}$ and $12 \mathrm{~cm}$ respectively using COTS RFID readers in a lab environment. The main idea is to construct a virtual antenna array in tag movement and leverage the statistical method to find the optimal trajectory. DAH is effective way to track and locate moving tags than previous work. However, thermal noise and external inference, perhaps from human moving besides RFID tags in actual circumstance will both affect the reported RF phase. Also, as the surveillance area expands, the computations will jeopardize the real-time performance. In addition, the accuracy of tracking unpredictable movements should be improved in some extent.

In this paper, we propose a novel positioning and tracking method based on measured phase using COTS RFID devices, called TrackT. The phase periodicity is mainly used in our approach to improve the ability of noise tolerance and real-time performance in tracking mobile tags. At first glance, the phase periodicity is a negative factor and many researchers try to eliminate the unknown parameter in previous work. However, the differences of the phase periodicity between two continuous reads are an effective indicator introduced in the paper, with stronger noise-tolerance than phase-difference method like DAH. The basic idea of TrackT is described as follows. We first divide the surveillance region into mm-level square grids and assume that there is a visual tag as same as the tracked tag on the center of every grid. (i) Known Movement. We compare the sequence of the difference of phase periodicity between each virtual tag and the physical one to acquire several candidate points and then separately compute the corresponding double difference of true phase to find the minimum value. So the grid with the minimum value is chosen as the optimal position of the tracked tag. Once the initial position has been determined, the predefined track will also be fitted. (ii) Unknown Movement. In this case, both the initial position and the tag's displacements should be estimated. We suppose that all of antennas read the tag at the same time, and then the tag's displacement every read round could be calculated using first-order Taylor series expansion. For the unknown initial position, we leverage the same method of tracking known movement trajectory to select

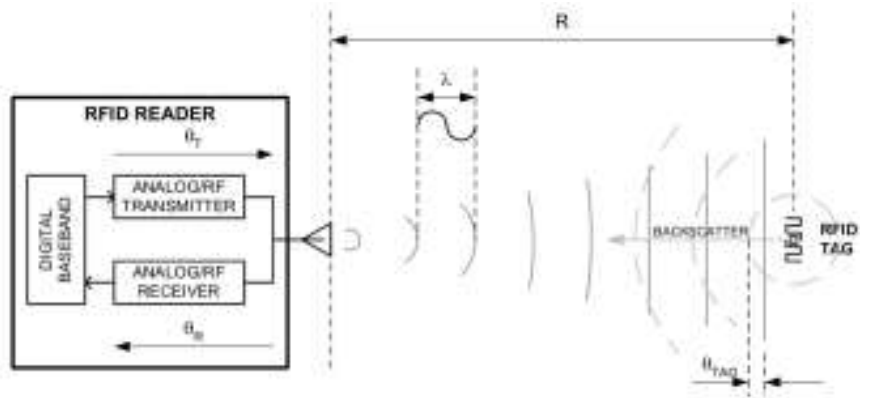

Fig. 1. Overview of RFID system. It shows a conceptual diagram of the radio wave propagation between an RFID reader and a passive RFID tag.

the optimal one. For achieving real-time performance, a rule to reduce the computations is also proposed in the paper.

Summary of Results: We build the system using Impinj R420 RFID reader, four 8dBi antennas and RFID passive tags. Our main results are described as follows:

(i) When four RFID antennas are deployed around the monitoring area, TrackT can achieve mm-level accuracy with mean error distances of $0.26 \mathrm{~cm}$ and $0.55 \mathrm{~cm}$ for controllable and uncontrollable movements.

(ii) TrackT is a real-time localization system so the computation time for producing an intermediate result should be less than the time interval of about $33 \mathrm{~ms}$ between successive inventories of the same tag. After optimization, TrackT can achieve a mean of about $11.21 \mathrm{~ms}$ computation time, which can meet the real-time requirement.

Contributions: TrackT can capture the known and unknown movement trajectories of mobile RFID tags with mm-level accuracy. As far as we know, TrackT is the first to leverage the phase periodicity with high noise tolerance to track mobile tags. Besides, we also exploit the localization theory of carrier phase measurement in global positioning system (GPS) to track unknown trajectory of the moving RFID tag. As a result, TrackT with less positioning error and higher real-time performance can be easily implemented in real applications based on COTS RFID devices.

The remainder of the paper is described as follows. The background and empirical studies are introduced in Section II. The main design of the TrackT approach is presented in Section III. We discuss additional details in Section IV. The implementation and evaluation are given in Section V. Finally, Section VI introduces the limitations and concludes the paper.

\section{PRELIMINARIES}

In this section, we mainly introduce RF phase, Doppler frequency shift, session persistence and phase periodicity. 


\section{A. RF Phase}

For an RF carrier wave at frequency $f(\mathrm{~Hz})$, the relation between frequency $f$ and wavelength $\lambda$ is given by

$$
\lambda=\frac{c}{f} \text { (meters) }
$$

where in air, the speed $\mathrm{c}$ of the electromagnetic wave is equal to the speed of light, i.e., $3 \times 10^{8} \mathrm{~m} / \mathrm{s}$. In China, a typical ultra-high frequency (UHF) reader has 16 channels working at 920 925 MHz industrial scientific medical (ISM) band.

As shown in Fig. 1, the distance between an RFID reader antenna and a passive RFID tag is R. In addition to the RF phase over distance, RFID hardware instruments including the reader's transmit circuits, the tag's reflection characteristic, and the reader's receiver circuits will all introduce some additional phase shifts $\varphi_{T}, \varphi_{T a g}$ and $\varphi_{R}$ respectively [12]. So the true phase $\Phi$ can be expressed as

$$
\Phi=\varphi+N=2 \pi \times \frac{2 R}{\lambda}+\varphi_{T}+\varphi_{R}+\varphi_{\text {Tag }}
$$

where $\varphi$, the output parameter from the RFID reader, is the measured phase within $[0,2 \pi]$. The unknown parameter $N$, an integral multiple of $2 \pi$, is called phase periodicity.

\section{B. Doppler Frequency Shift}

Doppler frequency shift is the shift in frequency of the received signal at the reader due to relative motion between the reader and the tag. Assume that the carrier frequency is $f$ and the signal frequency caused by the Doppler effect is $f_{r}$, so the Doppler frequency shift is defined as follows:

$$
f-f_{r}=\frac{2 f}{c} \frac{d \rho}{d t}
$$

where $\frac{d \rho}{d t}$ is the radial velocity with which the tracked tag moves away from or approaches the antenna. The factor of 2 arises from the tag backscattering the reader's carrier signal.

So the phase shift $n$ caused by Doppler effect is:

$$
\begin{aligned}
n & =2 \pi \times \int_{t}^{t+\Delta T}\left(f-f_{r}\right) d t \\
& =2 \pi \int_{t}^{t+\Delta T} \frac{2 f}{c} \frac{d \rho}{d t} d t \\
& =\frac{4 \pi\left(\rho_{t+\Delta T}-\rho_{t}\right)}{\lambda}
\end{aligned}
$$

where $\rho_{t+\Delta T}$ and $\rho_{t}$ are the distances from the tracked tag to the antenna at the time $t+\Delta T$ and $t$ respectively. $\Delta T$ denotes the time duration of a packet related to EPC length and reverses link rate. If $\rho_{t+\Delta T}>\rho_{t}$, the tag moves away from the antenna, i.e., $n>0$. If $\rho_{t+\Delta T}<\rho_{t}$, the tag moves close to the antenna, i.e., $n<0$. This feature is often used to determine tags in motion versus stationary tags and tag motion directionality.

Considering Doppler effect, the true phase is denoted by $\Phi=\varphi+N+n$. In COTS RFID readers, the slower reader inventory modes (e.g. Dense Reader, $\mathrm{M}=8$ ) should be configured to measure Doppler frequency shift because longer measurement intervals typically provide more accurate Doppler frequency shift estimates. But in this paper, the reader mode with higher read rate (e.g. Max Throughput) is used in our proposed method. In this case, the accuracy of the reported Doppler frequency shifts will be reduced significantly. Fortunately, since $\Delta T$ is generally at millisecond level for the low-speed RFID tags, it is reasonable for us to ignore the impact of Doppler effect on the true phase in the following.

\section{Session Persistence}

There are two inventory flags of ' $\mathrm{A}$ ' and 'B' for a tag. All inventory flags default to ' $A$ ' when a tag powers up and the flag ' $\mathrm{B}$ ' is the state when tag power is lost. Regardless of the state of ' $A$ ' and ' $B$ ', tags both allow inventory operations. The persistence of the state ' $\mathrm{B}$ ' is related to the inventory flag configured by RFID reader and tag power state. Indeed, there are four search modes in RFID reader, i.e., Dual Target Session 0 (Persistence time $\mathrm{T}_{\mathrm{S}}=0 \mathrm{~s}$ ), Single Target Session 1 $\left(\mathrm{T}_{\mathrm{S}}=0.5 \sim 5 \mathrm{~s}\right)$, Single Target Session 2 or $3\left(\mathrm{~T}_{\mathrm{S}}=2 \sim 60 \mathrm{~s}\right)$ and Single Target with Suppression $\left(\mathrm{T}_{\mathrm{s}}=0.5 \sim 5 \mathrm{~s}\right)$. For Dual Target mode, the state of the Session 0 flag persists indefinitely when tag power is on, but the state of the flag is lost immediately when tag power is lost. This session mode enables the reader to read tags continuously, which is suitable for read small batch of products in static environment. And Single Target mode has a fixed and limited persistence even when tag power is on; if the flag has not been refreshed by an inventory operation in more than the persistence time. This model is suitable for read large products in dynamic environment [20]. Due to the influence of multipath propagation and tag population size, the persistence of state ' $\mathrm{B}$ ' is uncertain for RFID reader, meaning that the time between successive inventories of the same tag is random. In summary, a tag can be interrogated for about 30 times per second under the read mode of the Session 0 flag [21], so the search mode of Dual Target Session 0 is employed in our proposed method.

\section{Phase Periodicity}

Suppose that two successive time-phase pairs are measured for the same tag, i.e., $\left(t_{i}, \varphi_{i}\right)$ and $\left(t_{i+1}, \varphi_{i+1}\right)$. In this case, the true phase are $\Phi_{i}=\varphi_{i}+N_{i}$ and $\Phi_{i+1}=\varphi_{i+1}+N_{i+1}$, and the Euclidean distances between the RFID tag and the antenna are $\rho_{i}$ and $\rho_{i+1}$ respectively. We also assume that $d_{i}$ represents the tag movement distance between $t_{i}$ and $t_{i+1}$. Since the system suffers from serious packet loss when the tag moves with a high speed, resulting that the tag even cannot be interrogated [22], we consider the case where tags move with a low speed in our system. In addition, the interval between successive inventories of the same tag is short enough, so it is feasible to assume $d_{i} \leq \lambda / 4$. Based on the triangle constraint, we have $0<\left|\rho_{i+1}-\rho_{i}\right|<d_{i}$, i.e.,

$$
\begin{aligned}
& \left|\frac{4 \pi}{\lambda}\left(\rho_{i+1}-\rho_{i}\right)\right|=\left|\Phi_{i+1}-\Phi_{i}\right| \\
& =\left|\left(\varphi_{i+1}-\varphi_{i}\right)+\left(N_{i+1}-N_{i}\right)\right|<\pi
\end{aligned}
$$

Since $N_{i}$ is an integral multiple of $2 \pi$, the difference of 
phase periodicity between two continuous reads, $\Delta N_{i}$, can be denoted as

$$
\Delta N_{i}=N_{i+1}-N_{i}=\left\{\begin{array}{cc}
0 & \left|\varphi_{i+1}-\varphi_{i}\right|<\pi \\
-2 \pi & \pi \leq \varphi_{i+1}-\varphi_{i} \leq 2 \pi \\
2 \pi & -2 \pi \leq \varphi_{i+1}-\varphi_{i} \leq-\pi
\end{array}\right.
$$

Note that the time between successive inventories of the same tag will depend on reader mode, tag population size, and environmental conditions (e.g. interference levels). While the RFID reader mode is set to the highest read rate, the minimum update interval is about 0.5 seconds for the population of 512 RFID tags in the read area. The tag population size in the read range should be considered to make sure $d_{i} \leq \lambda / 4$ in practice. Many methods [23],[24] are proposed to improve the time-efficiency for large-scale RFID systems.

\section{TRACKT OVERVIEW}

In this section, we give details on our tracking approach in two cases. In 2D scenario, a tracked RFID tag is aligned with the center of antennas. We first introduce how to track a mobile RFID tag's trajectory in the case of conveyor with known track that the trajectory function and the tag's speed are known for us before tracking. Then we describe how to track the unpredictable movement that both trajectory function and speed are unknown in prior.

\section{A. Movement with known track}

Assume that the phase sequence read by an RFID reader in tag's movement is $\varphi=\left\{\varphi_{1}, \varphi_{2}, \varphi_{3}, \ldots, \varphi_{n}\right\}$. While the tag moves along different trajectories in the monitoring area, the sequence $\varphi$ is also different from each other. In this case, the tag movement trajectory could be captured by distinguishing the corresponding phase sequence. However, it is very difficult to collect all of phase sequence generated by different trajectories for a tag moving in the region. In addition, the reported phase is suffered from the effect of the hardware noise and external interference, and the time between the two continuous inventories is random even on the same movement trajectory. In summary, there are above limitations to capture the tag's movement trajectory by comparing different phase sequences.

The sequence of the difference of phase periodicity is $\Delta N=\left\{\Delta N_{1}, \Delta N_{2}, \Delta N_{3}, \ldots, \Delta N_{n-1}\right\}$ corresponding to $\varphi$ based on (6). In our experiment, the measured phase follows a typical Gaussian distribution with a standard deviation of 0.1 radians, then $\Delta \varphi_{i}=\left(\varphi_{i+1}-\varphi_{i}\right) \sim N(0,0.1 \times \sqrt{2})$, but the slight variation of phase estimates has little impact on $\Delta N$. For example, due to $\Delta \varphi_{i}=1.2$ radians and $\Delta \varphi_{i}=2.5$ radians, their corresponding $\Delta N$ are both equal to 0 . So we consider that the difference of phase periodicity is not sensitive to noise interference. Moreover, unlike the phase values varying within $[0,2 \pi], \Delta N_{i}$ has only three certain values, i.e., 0 and $\pm 2 \pi$.

Therefore, the sequence of the difference of phase periodicity is employed to track the tag's trajectory and locate the initial position in this paper.

1) Preprocessing

In the 2D scenario of RFID tag's movement with known track, the tracked target attached on an RFID tag moves with a constant speed. RFID antennas are deployed around the surveillance region to read the tag for tracking and positioning. At present, the most common uniform tracks are a variety of conveyor belts, including uniform linear and circular track. Conveyors are widely used for moving and sorting goods packaged in cartons, boxes, or shrink-wrapped containers. If we can know the initial position on the track, the following trajectory can be determined. Based on this observation, we first make the following assumptions:

(i) Suppose that the tag with uniform motion in the surveillance region keeps in the stationary state, so each of fixed antennas uniformly moves along the opposite direction relative to the tag.

(ii) The surveillance region is divided into $W \times L$ square grids at mm-level (less than $1 \mathrm{~cm}$ ) and suppose that there is a virtual tag as the same as the tracked one on the center of each grid.

2) Comparison rule

In the rectangular coordinate system, let the initial coordinate of the tag be $\left(X\left(T_{1}\right), Y\left(T_{1}\right)\right)$. Suppose that there are m reader antennas deployed around the surveillance region to read the tracked tag. Once receiving $\mathrm{n}$ reads for each antenna, TrackT will calculate the initial position of the moving tag. The $\mathrm{j}^{\text {th }}$ read time and phase value measured by the $\mathrm{i}^{\text {th }}$ antenna are $t\left(A_{\mathrm{i}, \mathrm{j}}\right)$ and $\varphi\left(A_{\mathrm{i}, \mathrm{j}}\right)$ respectively. The difference of phase periodicity is denoted by $\Delta N\left(A_{\mathrm{i}, \mathrm{j}}\right)$. In addition, as the tracked tag moves, we assume that reader antennas move along the opposite direction, so the virtual position of the $i^{\text {th }}$ antenna is $A_{\mathrm{i}, \mathrm{j}}=A_{\mathrm{i}, 1}+\overrightarrow{\mathrm{v}} \times\left[t\left(A_{\mathrm{i}, \mathrm{j}}\right)-t\left(A_{\mathrm{i}, 1}\right)\right]$, where $\overrightarrow{\mathrm{v}}$ represents the speed function of the mobile tag and $A_{\mathrm{i}, 1}$ is the coordinate of the $\mathrm{i}^{\text {th }}$ fixed antenna, i.e., $A_{\mathrm{i}, 1}=\left(X\left(A_{\mathrm{i}, 1}\right), Y\left(A_{\mathrm{i}, 1}\right)\right)$.

Definition $1 \varphi\left(G_{W, L}, A_{\mathrm{i}, \mathrm{j}}\right)$ is the $\mathrm{j}^{\text {th }}$ theoretical phase value of the virtual tag on the grid $G_{W, L}$ read by the $\mathrm{i}^{\text {th }}$ antenna. Ignoring the additional phase $\varphi_{T}, \varphi_{T a g}$ and $\varphi_{R}$, the theoretical phase is defined as follows:

$$
\varphi\left(G_{W, L}, A_{\mathrm{i}, \mathrm{j}}\right)=\frac{4 \pi}{\lambda}\left|G_{W, L} \cdot A_{\mathrm{i}, \mathrm{j}}\right| \bmod 2 \pi
$$

where $\left|G_{W, L} \cdot A_{\mathrm{i}, \mathrm{j}}\right|$ is the Euclidean distance between the grid $G_{W, L}$ and the virtual antenna $A_{\mathrm{i}, \mathrm{j}}$. Also, the symbol "mod" means the modulo operation because the reported phase is a periodic function within $[0,2 \pi]$.

Definition $2 \Delta N\left(G_{W, L}, A_{\mathrm{i}, \mathrm{j}}\right)$ is the difference value of phase periodicity between $\varphi\left(G_{W, L}, A_{\mathrm{i}, \mathrm{j}}\right)$ and $\varphi\left(G_{W, L}, A_{\mathrm{i}, \mathrm{j}+1}\right)$ : 


$$
\Delta N\left(G_{W, L}, A_{\mathrm{i}, \mathrm{j}}\right)=\left\{\begin{array}{cc}
0 & \left|\varphi\left(G_{W, L}, A_{\mathrm{i}, j+1}\right)-\varphi\left(G_{W, L}, A_{\mathrm{i}, \mathrm{j}}\right)\right|<\pi \\
-2 \pi & \pi \leq \varphi\left(G_{W, L}, A_{\mathrm{i}, \mathrm{j}+1}\right)-\varphi\left(G_{W, L}, A_{\mathrm{i}, \mathrm{j}}\right) \leq 2 \pi \\
2 \pi & -2 \pi \leq \varphi\left(G_{W, L}, A_{\mathrm{i}, \mathrm{j}+1}\right)-\varphi\left(G_{W, L}, A_{\mathrm{i}, \mathrm{j}}\right) \leq-\pi
\end{array}\right.
$$

Definition $3 \operatorname{Num}\left(G_{W, L}\right)$ is called the position correlation between the virtual tag on the grid $G_{W, L}$ and the tracked tag, which is defined as follows:

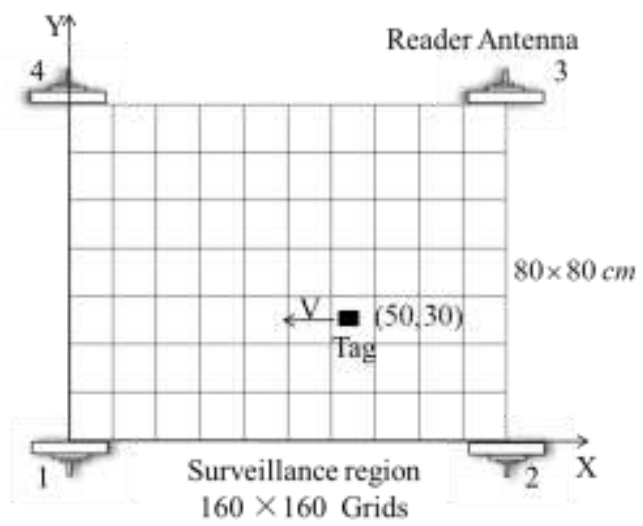

Fig. 2. Surveillance region. The surveillance region of $80 \mathrm{~cm} \times 80 \mathrm{~cm}$ is divided into the number of $160 \times 160$ square grids with the width of $0.5 \mathrm{~cm}$ and then mapped into the coordinate system. The XY-axis units are both centimeters. The antennas $1-4$ are respectively deployed on $(0,0),(110,0)$, $(100,110)$ and $(0,110)$. The tracked tag moves along a known track from the initial position of $(50,30)$.

$$
\operatorname{Num}\left(G_{W, L}\right)=\sum_{i=1}^{m} \sum_{j=1}^{n-1} f\left[\Delta N\left(A_{\mathrm{i}, \mathrm{j}}\right), \Delta N\left(G_{W, L}, A_{\mathrm{i}, \mathrm{j}}\right)\right]
$$

where $\mathrm{m}$ is the number of antennas, $\mathrm{n}$ is the read times in a read round for each antenna and $f\left[\Delta N\left(A_{\mathrm{i}, \mathrm{j}}\right), \Delta N\left(G_{W, L}, A_{\mathrm{i}, \mathrm{j}}\right)\right]$ is the position correlation function between $\Delta N\left(\mathrm{~A}_{\mathrm{i}, \mathrm{j}}\right)$ and $\Delta N\left(G_{W, L}, A_{\mathrm{i}, \mathrm{j}}\right)$ :

$f\left[\Delta N\left(A_{\mathrm{i}, \mathrm{j}}\right), \Delta N\left(G_{W, L}, A_{\mathrm{i}, \mathrm{j}}\right)\right]= \begin{cases}1 & \Delta N\left(A_{\mathrm{i}, \mathrm{j}}\right)=\Delta N\left(G_{W, L}, A_{\mathrm{i}, \mathrm{j}}\right) \\ 0 & \Delta N\left(A_{\mathrm{i}, \mathrm{j}}\right) \neq \Delta N\left(G_{W, L}, A_{\mathrm{i}, \mathrm{j}}\right)\end{cases}$

The smaller the distance of the tracked tag to the virtual tag on the grid is, the larger the value of $\mathrm{Num}_{W, \mathrm{~L}}$ is. In this case, the grid with the maximum $\operatorname{Num}\left(G_{W, L}\right)$ could be regarded as the optimal position of the tracked tag.

To visually understand this idea, let us consider the simple example shown in Fig. 2. The range of surveillance region is $80 \mathrm{~cm} \times 80 \mathrm{~cm}$ composed by $160 \times 160$ square grids with the width of $0.5 \mathrm{~cm}$. The RFID reader antennas $1-4$ are deployed on both sides of surveillance region, where the coordinates of antennas $1-4$ are respectively $(0,0),(110,0),(100,110)$ and $(0,110)$ in unit of centimeter. The initial position of tag before the tag movement is $(50,30)$, moving along the negative direction of $\mathrm{x}$-axis with the speed of about $20 \mathrm{~cm} / \mathrm{s}$. We collect the about 300 phase values by all of antennas in 2.5 seconds, so the expected maximum of $N_{u m_{W, \mathrm{~L}}}$ will research 296. In Fig. 3 , the maximum point on the grid $\mathrm{W}=100, \mathrm{~L}=60$ is 286 , meaning that the position of the virtual tag on the grid is closest to the tracked tag. The corresponding coordinate is $(49.75,29.75)$, deviating from the ground truth about $0.3536 \mathrm{~cm}$. However, in our experiment we find that there may be multiple candidates as the number of collected phase data decreases. In Fig. 4, if we calculate $\operatorname{Num}\left(G_{W, L}\right)$ only using the phase from antennas 1 and 2, we have $\operatorname{Num}\left(G_{100,60}\right)=\operatorname{Num}\left(G_{65,53}\right)=144$. The corresponding coordinates are respectively $(49.75,29.75)$ and $(32.25,26.25)$, and the distance between the two coordinates is $17.85 \mathrm{~cm}$. So we believe that phase periodicity results in multiple maximums in the surveillance region.

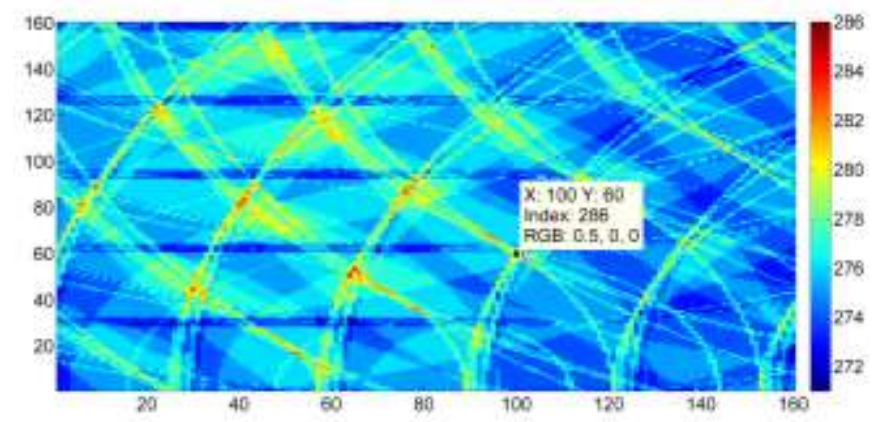

Fig. 3. The optimal position on the grid. The tracked tag locates potentially on the grid of $\mathrm{W}=100, \mathrm{~L}=60$ with the maximum position correlation.

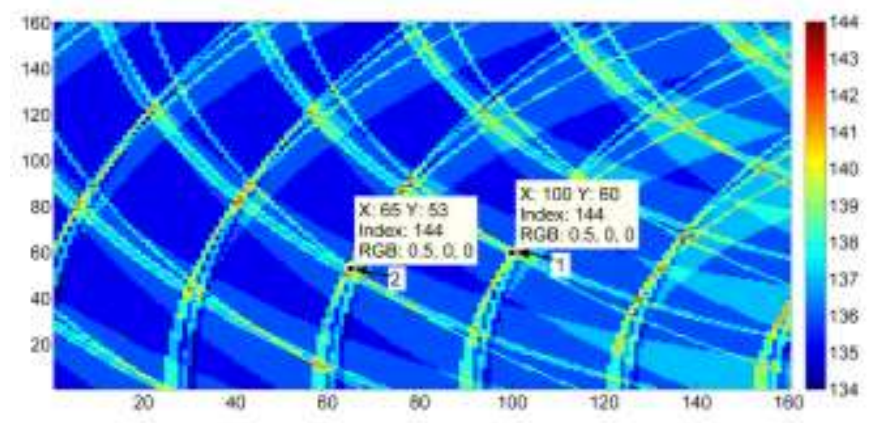

Fig. 4. Position ambiguity. With the decrease of collected phase data, there are two grids with maximum values, resulting in the position ambiguity of tracked tag.

\section{3) Differential true phase for position ambiguity}

In the following, we introduce how to eliminate the position ambiguity based on the true phase defined in Section II.

Definition $4 \Phi\left(G_{W, L}, A_{\mathrm{i}, \mathrm{j}}\right)$ is the $\mathrm{j}^{\text {th }}$ true phase of the virtual tag on the grid $G_{W, L}$ read by the $\mathrm{i}^{\text {th }}$ antenna. Here, we also ignore $\varphi_{T}, \varphi_{T a g}$ and $\varphi_{R}$, so the true phase with phase periodicity is denoted as follows:

$$
\Phi\left(G_{W, L}, A_{\mathrm{i}, \mathrm{j}}\right)=\frac{4 \pi}{\lambda}\left|G_{W, L} \cdot A_{\mathrm{i}, \mathrm{j}}\right|
$$

Definition $5 \Phi\left(A_{i, j}\right)$ is the $\mathrm{j}^{\text {th }}$ true phase of the tracked tag read by the $\mathrm{i}^{\text {th }}$ antenna:

$$
\Phi\left(A_{\mathrm{i}, \mathrm{j}}\right)=\varphi\left(A_{\mathrm{i}, \mathrm{j}}\right)+N\left(A_{\mathrm{i}, \mathrm{j}}\right)
$$

where $\varphi\left(A_{\mathrm{i}, \mathrm{j}}\right)$ is the reported phase value and $N\left(A_{\mathrm{i}, \mathrm{j}}\right)$ is the phase periodicity of the tracked tag.

Definition $6 \Delta \Phi\left(G_{W, L}\right)$ is called Single Difference, which is calculated by 
$\Delta \Phi\left(G_{W, L}\right)=\sum_{i=1}^{m} \sum_{j=1}^{n}\left|\Phi\left(G_{W, L}, A_{i, j}\right)-\Phi\left(A_{i, j}\right)\right|$

$=\sum_{i=1}^{m} \sum_{j=1}^{n}\left[\left[\varphi\left(G_{W, L}, A_{i, j}\right)+N\left(G_{W, L}, A_{i, j}\right)\right]-\left[\varphi\left(A_{i, j}\right)+N\left(A_{i, j}\right)\right]\right.$

where $N\left(A_{i, j}\right)$ is an unknown parameter for us and $N\left(G_{W, L}, A_{i, j}\right)$ is the phase periodicity of virtual tag on the grid $G_{\mathrm{W}, \mathrm{L}}$. Based on the known distance $\left|G_{W, L} \cdot A_{i, j}\right|$, we have $N\left(G_{W, L}, A_{i, j}\right)=\left(\frac{4 \pi}{\lambda}\left|G_{W, L} \cdot A_{i . j}\right| \backslash 2 \pi\right) \times 2 \pi$, where the symbol ' I' represents the quotient operator to obtain quotient of $\frac{4 \pi}{\lambda}\left|G_{W, L} \cdot A_{i, j}\right|$ and $2 \pi$.

Obviously, the closer the virtual tag on the grid is to the initial position of the tracked tag, the smaller the value of $\Delta \Phi_{W, \mathrm{~L}}$ is. Next we will take difference of the single difference to eliminate the unknown parameter $N_{A_{i, j}}$.

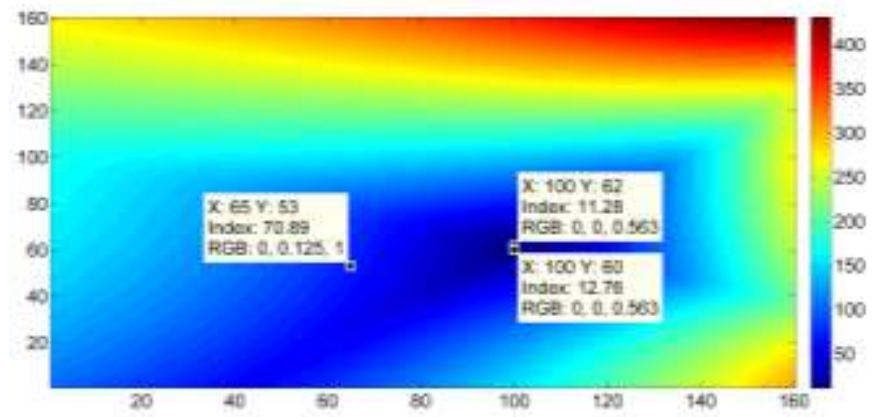

Fig. 5. The results of double difference. Compared with the values of double difference on the grids of $\mathrm{W}=100, \mathrm{~L}=60$ and $\mathrm{W}=65, \mathrm{~L}=53$ with same position correlation in Fig. 4, the optimal position is on the grid of $\mathrm{W}=100, \mathrm{~L}=60$ with the minimum value. And the positioning result of TrackT on the grid of $\mathrm{W}=100, \mathrm{~L}=60$ has a lower error distance than that of $\mathrm{DAH}$ on the grid of $\mathrm{W}=100, \mathrm{~L}=62$.

Definition 7 Diff $\Delta \Phi\left(G_{W, L}\right)$ is called Double Difference, which is calculated by:

$$
\operatorname{Diff} \Delta \Phi\left(G_{W, L}\right)=\sum_{i=1}^{m} \sum_{j=1}^{n-1}\left[\left[\Phi\left(G_{W, L}, A_{\mathrm{i}, \mathrm{j}}\right)-\Phi\left(A_{\mathrm{i}, \mathrm{j}}\right)\right]-\left.\left[\Phi\left(G_{W, L}, A_{\mathrm{i}, 1}\right)-\Phi\left(A_{\mathrm{i}, 1}\right)\right]\right|^{(14}\right.
$$

Hence, the initial coordinate can be calculated by the function $\mathrm{f}\left[X\left(T_{1}\right), Y\left(T_{1}\right)\right]=\min \left[\operatorname{Diff} \Delta \Phi\left(G_{W, L}\right)\right]$. And double difference can reduce the effect of measurement noise as much as possible, which can be proved as follows:

For the $\mathrm{j}^{\text {th }}$ and $\mathrm{k}^{\text {th }}$ phase values collected by the $\mathrm{i}^{\text {th }}$ antenna, we have

$$
\begin{aligned}
& \mid\left[\Phi\left(G_{W, L}, A_{i, \mathrm{j}}\right)-\Phi\left(A_{\mathrm{i}, \mathrm{j}}\right)\right]-\left[\Phi\left(G_{W, L}, A_{i, \mathrm{k}}\right)-\Phi\left(A_{\mathrm{i}, \mathrm{k}}\right)\right] \\
& =\mid\left[\Phi\left(G_{W, L}, A_{\mathrm{i}, \mathrm{j}}\right)-\Phi\left(G_{W, L}, A_{\mathrm{i}, \mathrm{k}}\right)\right]-\left[\Phi\left(A_{\mathrm{i}, \mathrm{j}}\right)-\Phi\left(A_{\mathrm{i}, \mathrm{k}}\right)\right] \\
& =\left|\frac{4 \pi}{\lambda}\left[\left|G_{W, L} \cdot A_{\mathrm{i}, \mathrm{j}}\right|-\left|G_{W, L} \cdot A_{\mathrm{i}, \mathrm{k}}\right|\right]-\left[\varphi\left(A_{\mathrm{i}, \mathrm{j}}\right)-\varphi\left(A_{\mathrm{i}, \mathrm{k}}\right)+N\left(A_{\mathrm{i}, \mathrm{j}}\right)-N\left(A_{\mathrm{i}, \mathrm{k}}\right)\right]\right|
\end{aligned}
$$

For the uniform linear track, as the tracked tag moves away from the initial position, we have:

$$
\max \left|N\left(A_{\mathrm{i}, \mathrm{j}}\right)-N\left(A_{\mathrm{i}, \mathrm{k}}\right)\right|=\left|N\left(A_{\mathrm{i}, \mathrm{j}}\right)-N\left(A_{\mathrm{i}, 1}\right)\right|
$$

Note that for the other motion trajectories, we also assume that $k=1$, although the value of $N\left(A_{\mathrm{i}, \mathrm{j}}\right)-N\left(A_{\mathrm{i}, 1}\right)$ will not be the maximum. Then $N\left(A_{\mathrm{i}, \mathrm{j}}\right)-N\left(A_{\mathrm{i}, 1}\right)$ can be expressed as:

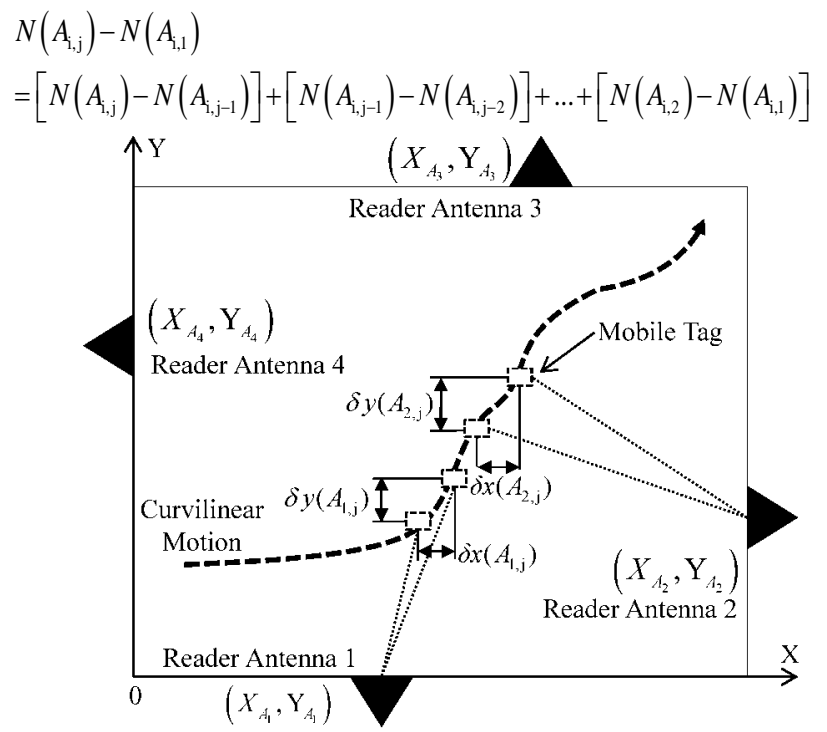

Fig. 6. Curvilinear motion. The RFID tag moves with unpredictable movement in the monitoring area.

Based on (6), we depend on the collected phase, $\left\{\varphi_{A_{i, 1}}, \varphi_{A_{i, 2}}, \ldots, \varphi_{A_{i, j}}\right\}$, to calculate the every difference value of $N\left(A_{\mathrm{i}, \mathrm{j}}\right)-N\left(A_{\mathrm{i}, 1}\right)$.

Because all of $\varphi\left(A_{\mathrm{i}, \mathrm{j}}\right)(j=1,2, \ldots)$ are independent standard normal random variables with the same distribution, i.e., $\varphi\left(A_{\mathrm{i}, \mathrm{j}}\right) \sim \mathrm{N}(0, \sigma)$ for the $\mathrm{i}^{\text {th }}$ antenna, we have $\left[\varphi\left(A_{\mathrm{i}, \mathrm{j}}\right)-\varphi\left(A_{\mathrm{i}, 1}\right)\right] \sim \mathrm{N}(0, \sqrt{2} \times \sigma)$. As described before, $\sigma=0.1$ radians, so the standard deviation of phase difference is 0.14 radians. However, since $\left|N\left(A_{\mathrm{i}, \mathrm{j}}\right)-N\left(A_{\mathrm{i}, 1}\right)\right|=2 k \pi$, the value will be far larger than 0.14 radians. In this case, the variance of phase values caused by thermal noise and external interference has little impact on the final result of $\operatorname{Diff} \Delta \Phi\left(G_{W, L}\right)$ for different antennas. In this way, TrackT has strong noise tolerance to multipath effect in practice.

Then we put all of candidates with the same position correlation into (14) to calculate the results of double difference, so the grid with the minimum value is considered as the final position of the tracked tag. In Fig. 4, there are two candidates on the grid of $\mathrm{W}=100, \mathrm{~L}=60$ and $\mathrm{W}=65, \mathrm{~L}=53$ and the corresponding values of double difference are respectively $\operatorname{Diff} \Delta \Phi\left(G_{100,60}\right)=12.76$ and $\operatorname{Diff} \Delta \Phi\left(G_{65,53}\right)=70.89$. The optimal position is obviously on the grid of $\mathrm{W}=100, \mathrm{~L}=60$ and the corresponding coordinate is $(49.75,29.75)$. The distance error deviates from the ground truth about $0.3536 \mathrm{~cm}$. In addition, there is a minimum value on the grid of $\mathrm{W}=100, \mathrm{~L}=62$ in the surveillance zone. The corresponding double difference is $\operatorname{Diff} \Delta \Phi\left(G_{100,62}\right)=11.28$, and the distance error is $0.7906 \mathrm{~cm}$. The positioning accuracy in TrackT has a lower 
error distance than that in DAH [19] based on only double difference, which will be verified in the Section V.

\section{B. Movement with unknown track}

As shown in Fig. 6, an RFID tag moves along the curvilinear track in 2D scenario. Similarly to the previous method of tracking the known trajectory, we also assume that each of antennas moves in the opposite direction of the tag movement. The time interval between the $j^{\text {th }}$ and $(j+1)^{\text {th }}$ inventories of the tracked tag read by the $\mathrm{i}^{\text {th }}$ antenna is $\Delta t\left(A_{i, j}\right)$, so during the time interval, horizontal and vertical displacements of the $i^{\text {th }}$ antenna relative to the tag can be respectively represented as $\delta x\left(A_{i, j}\right)$ and $\delta y\left(A_{i, j}\right)$, which is subject to $\max \left(\sqrt{\left[\delta x\left(A_{i, j}\right)\right]^{2}+\left[\delta y\left(A_{i, j}\right)\right]^{2}}\right) \leq \frac{\lambda}{4}$.

RFID employs a slotted-aloha media access scheme, which means that the order in which tags are inventoried will be random [20],[25], so antennas can't read the tag at the same time. Suppose that in every round each antenna reads the tag one time. The Euclidean distance of the tracked tag to the $\mathrm{i}^{\text {th }}$ antenna is $\rho\left(A_{i, j}\right)$ at the time $t\left(A_{i, j}\right)$. Based on the relationship between the true phase $\Phi\left(A_{\mathrm{i}, \mathrm{j}}\right)$ and the distance of the tag to $\mathrm{i}^{\text {th }}$ antenna, we have

$$
\begin{aligned}
& \rho\left(A_{i, j}\right)=\frac{\lambda}{4 \pi}\left[\varphi\left(A_{i, j}\right)+N\left(A_{i, j}\right)\right] \\
& =\sqrt{\left[X\left(T_{1}\right)-X\left(A_{i, j}\right)\right]^{2}+\left[Y\left(T_{1}\right)-Y\left(A_{i, j}\right)\right]^{2}}
\end{aligned}
$$

where $\left(X\left(T_{1}\right), Y\left(T_{1}\right)\right)$ is the unknown initial coordinate of the tracked tag in the curvilinear motion and $\left(X\left(A_{i, j}\right), Y\left(A_{i, j}\right)\right)$ is the coordinate of the $\mathrm{i}^{\text {th }}$ virtual antenna at the time $t\left(A_{i, j}\right)$.

A first-order Taylor series approximation can make unsolvable problems possible for a restricted domain [26]. So we compute a first-order Taylor series expansion around the point $\left(X\left(A_{i, j}\right), Y\left(A_{i, j}\right)\right)$ of the function $\rho\left(A_{i, j}\right)$ :

$$
\begin{aligned}
& \rho\left(A_{i, j}\right)=\sqrt{\left[X\left(T_{1}\right)-X\left(A_{i, j}\right)\right]^{2}+\left[Y\left(T_{1}\right)-Y\left(A_{i, j}\right)\right]^{2}} \\
& \approx \rho\left(A_{i, j-1}\right)+\frac{X\left(T_{1}\right)-X\left(A_{i, j-1}\right)}{\rho\left(A_{i, j-1}\right)} \delta x\left(A_{i, j-1}\right)+\frac{Y\left(T_{1}\right)-Y\left(A_{i, j-1}\right)}{\rho\left(A_{i, j-1}\right)} \delta y\left(A_{i, j-1}\right)
\end{aligned}
$$

where the coordinate point of the $\mathrm{i}^{\text {th }}$ virtual antenna at the time $t\left(A_{i, j}\right)$ can be denoted by

$$
\left\{\begin{array}{l}
X\left(A_{i, j}\right)=X\left(A_{i, j-1}\right)+\delta x\left(A_{i, j-1}\right) \\
Y\left(A_{i, j}\right)=Y\left(A_{i, j-1}\right)+\delta y\left(A_{i, j-1}\right)
\end{array}\right.
$$

For $\rho\left(A_{i, j-1}\right)$ and $\rho\left(A_{i, j}\right)$, we have

$$
\begin{aligned}
& \rho\left(A_{i, j}\right)-\rho\left(A_{i, j-1}\right) \\
& =\frac{\lambda}{4 \pi}\left[\varphi\left(A_{i, j}\right)-\varphi\left(A_{i, j-1}\right)+N\left(A_{i, j}\right)-N\left(A_{i, j-1}\right)\right] \\
& =\sqrt{\left[X\left(T_{1}\right)-X\left(A_{i, j-1}\right)\right]^{2}+\left[Y\left(T_{1}\right)-Y\left(A_{i, j-1}\right)\right]^{2}}-\rho\left(A_{i, j-1}\right) \\
& \approx \frac{X\left(T_{1}\right)-X\left(A_{i, j-1}\right)}{\rho\left(A_{i, j-1}\right)} \delta x\left(A_{i, j-1}\right)+\frac{Y\left(T_{1}\right)-Y\left(A_{i, j-1}\right)}{\rho\left(A_{i, j-1}\right)} \delta y\left(A_{i, j-1}\right)
\end{aligned}
$$

Thus,

$\frac{\lambda}{4 \pi}\left[\varphi\left(A_{i, j}\right)-\varphi\left(A_{i, j-1}\right)+N\left(A_{i, j}\right)-N\left(A_{i, j-1}\right)\right]$

$\approx \frac{X\left(T_{1}\right)-X\left(A_{i, j-1}\right)}{\rho\left(A_{i, j-1}\right)} \delta x\left(A_{i, j-1}\right)+\frac{Y\left(T_{1}\right)-Y\left(A_{i, j-1}\right)}{\rho\left(A_{i, j-1}\right)} \delta y\left(A_{i, j-1}\right)$

In our experiment, we deploy 4 directional antennas to acquire phase data for tracking a mobile tag accurately. Since in a round the read time among different antennas are very close to each other, about $33 \mathrm{~ms}$, it is reasonable for us to assume that each antenna reads the measured phase at the same time, which means the tag displacements measured by 4 antennas between successive read rounds are the same, i.e., $\delta x\left(A_{i, j-1}\right)=\delta x\left(A_{j-1}\right)$ and $\delta y\left(A_{i, j-1}\right)=\delta y\left(A_{j-1}\right)$. Based on phase data collected by 4 antennas, the equation (21) can be denoted in matrix form, i.e.,

$$
A x=B
$$

where

$$
\begin{aligned}
& A=\left[\begin{array}{cc}
\frac{X\left(T_{1}\right)-X\left(A_{1, j-1}\right)}{\rho\left(A_{1, j-1}\right)} & \frac{Y\left(T_{1}\right)-Y\left(A_{1, j-1}\right)}{\rho\left(A_{1, j-1}\right)} \\
\frac{X\left(T_{1}\right)-X\left(A_{2, j-1}\right)}{\rho\left(A_{2, j-1}\right)} & \frac{Y\left(T_{1}\right)-Y\left(A_{2, j-1}\right)}{\rho\left(A_{2, j-1}\right)} \\
\frac{X\left(T_{1}\right)-X\left(A_{3, j-1}\right)}{\rho\left(A_{3, j-1}\right)} & \frac{Y\left(T_{1}\right)-Y\left(A_{3, j-1}\right)}{\rho\left(A_{3, j-1}\right)} \\
\frac{X\left(T_{1}\right)-X\left(A_{4, j-1}\right)}{\rho\left(A_{4, j-1}\right)} & \frac{Y\left(T_{1}\right)-Y\left(A_{4, j-1}\right)}{\rho\left(A_{4, j-1}\right)}
\end{array}\right], \\
& B=\frac{\lambda}{4 \pi}\left[\begin{array}{c}
\varphi\left(A_{1, j}\right)-\varphi\left(A_{1, j-1}\right)+N\left(A_{1, j}\right)-N\left(A_{1, j-1}\right) \\
\varphi\left(A_{2, j}\right)-\varphi\left(A_{2, j-1}\right)+N\left(A_{2, j}\right)-N\left(A_{2, j-1}\right) \\
\varphi\left(A_{3, j}\right)-\varphi\left(A_{3, j-1}\right)+N\left(A_{3, j}\right)-N\left(A_{3, j-1}\right) \\
\varphi\left(A_{4, j}\right)-\varphi\left(A_{4, j-1}\right)+N\left(A_{4, j}\right)-N\left(A_{4, j-1}\right)
\end{array}\right], \\
& x=\left[\delta x\left(A_{j-1}\right) \delta y\left(A_{j-1}\right)\right]^{\mathrm{T}},(j \geq 2)
\end{aligned}
$$

The coordinate $(0,0)$ is firstly chosen as the initial point of the tracked tag. We can estimate the displacement $x$ of virtual antennas using least squares [27], [28]. After enough displacements are generated, the unknown initial coordinate $\left(X\left(T_{1}\right), Y\left(T_{1}\right)\right)$ can be calculated by acquiring the maximum values of the position correlation $\operatorname{Num}\left(G_{W, L}\right)$ and eliminating the position ambiguity as the same process as tracking the known trajectory of a mobile tag. In addition, since the virtual antennas move in the opposite direction to the tracked tag, the horizontal and vertical displacements of the tag in practice are 
$\delta x\left(T_{j-1}\right)=-\delta x\left(A_{j-1}\right)$ and $\delta y\left(T_{j-1}\right)=-\delta y\left(A_{j-1}\right)$. So a completed trajectory of unknown movement is fitted from the above steps.

\section{DISCUSSION}

In this section, we attempt to answer some practical issues.

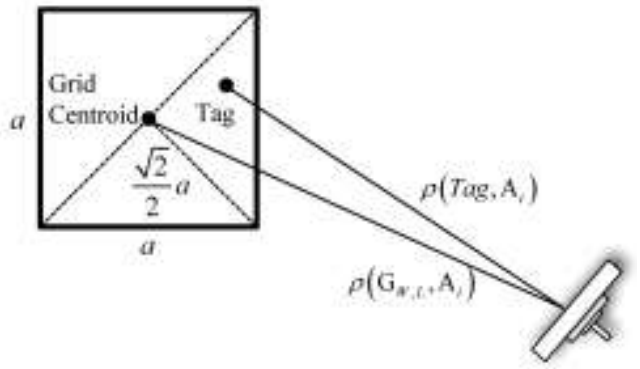

Fig.7. The rule of reducing computations. If the tracked tag maybe on the grid, the distance difference between $\rho\left(\mathrm{G}_{W, L}, A_{i}\right)$ and $\rho\left(T a g, A_{i}\right)$ should be less than $\frac{\sqrt{2}}{2} a$

\section{A. How to reduce the computations in TrackT?}

We know that the read range of a reader's antenna is generally about $10 \mathrm{~m}$, so the surveillance region composed of multiple antennas is so large enough that the number of grids at $\mathrm{mm}$-level in the region is also huge, which will jeopardize the real-time tracking capability.

From Fig. 3 and 4, the value of the deeper blue grids is smaller than others. That is to say, the ground truth of tracked tag doesn't exist on these grids so the related computation is unnecessary for us. As shown in Fig. 7, assume that the grid width is $a$, the distance from the center of grid to four angles is $\frac{\sqrt{2}}{2} a$ and the distances from the grid centroid and the position of tracked tag to the $\mathrm{i}^{\text {th }}$ antenna are respectively $\rho\left(G_{W, L}, A_{i}\right)$ and $\rho\left(\operatorname{Tag}, A_{i}\right)$. If the tracked tag is on the grid, then $\left|\rho\left(\mathrm{G}_{W, L}, A_{i}\right)-\rho\left(\operatorname{Tag}, A_{i}\right)\right| \leq \frac{\sqrt{2}}{2} a$. The measured phase of the tracked tag by the $\mathrm{i}^{\text {th }}$ antenna $A_{i}$ is $\varphi\left(A_{i}\right)$. Ignoring the phase periodicity, the phase of the virtual tag is $\varphi\left(G_{W, L}, A_{\mathrm{i}}\right)=\frac{4 \pi \rho\left(G_{W, L}, A_{i}\right)}{\lambda} \bmod 2 \pi$. If the tracked tag is on the grid $G_{W, L}$, then $\left|\frac{\lambda}{4 \pi}\left[\varphi\left(G_{W, L}, A_{\mathrm{i}}\right)-\varphi\left(A_{i}\right)\right]\right| \leq \frac{\sqrt{2}}{2} a$. So we only need to compute the position correlation $\operatorname{Num}\left(G_{W, L}\right)$ of the grid meeting the above limitation. Our evaluation shows that $65 \%$ of computation will be reduced using this method.

In addition, the limitation will not affect the positioning accuracy. For example, in Fig. 3 we calculate $\operatorname{Num}\left(G_{W, L}\right)$ between virtual tags on the every grid and the tracked tag. Here, we use above limitation to reduce computations and the result is shown in Fig. 8. A majority of non-candidate grids can be ignored because the values of $\operatorname{Num}\left(G_{W, L}\right)$ for many grids are equal to zero. And the maximum value of $\operatorname{Num}\left(G_{W, L}\right)$ is equal to 202 on the grid of $\mathrm{W}=101, \mathrm{~L}=60$. The corresponding coordinate is $(50.25,29.75)$ and the distance error deviates from the ground truth about $0.3536 \mathrm{~cm}$. As a result, the optimization method to reduce the computations will be employed in the following experiment.

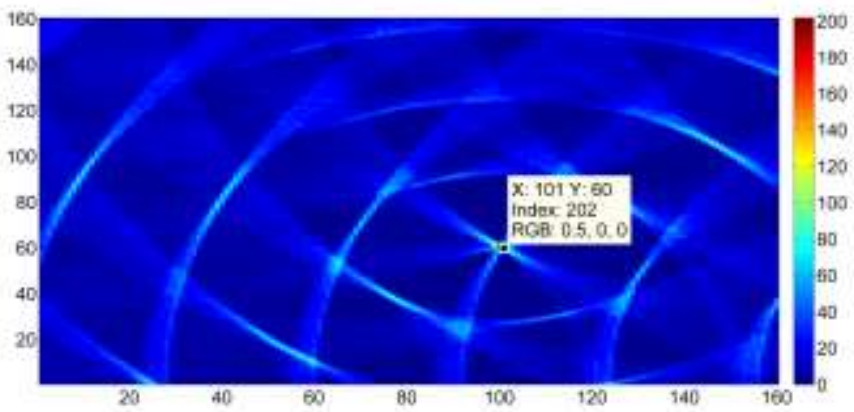

Fig. 8. The optimized result Compared with Fig. 3, the positioning results before and after optimization have the same distance error deviated from the ground truth.

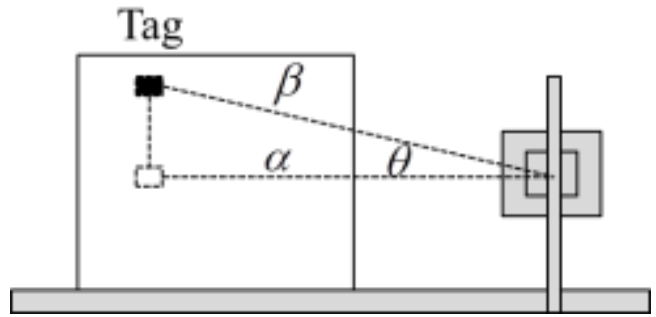

Fig. 9. 3D space. The tag and the center of antenna aren't on the same plane. In this case, the surveillance plane is composed of cubes with mm-level width, which will be inevitable to increase computations.

\section{B. How to work with Multipath effect and Non-Line of Sight?}

Multipath effect is a common issue for wireless localization systems. Undesired signals in the actual environment can combine with the primary backscatter, thereby increasing or decreasing the received signal power at the antenna receiver. The signal propagation along Non-Line of Sight (NLOS) introduces multipath effects to challenge the accuracy in phase measurements.

TrackT works with multipath effect from three aspects. At first, the difference values of phase periodicity with strong noise tolerance are mainly leveraged in TrackT to locate RFID tags. TrackT finds the optimal trajectory of the mobile tag by multiple reads. Second, the tag's mobility can help antennas read phase data from different directions. Lots of measurements from various directions can reduce the influence of multipath effect. Third, there are generally 16 frequency channels for an RFID reader. We can select the most sensitive frequency to the tag in order to reduce the impact of multipath propagation. Currently, there have been many excellent solutions [29], [30] in recent years. Their basic ideas are to find out the direct path among all paths.

\section{How to handle with $3 D$ scenario?}

In practice, it is impossible that all of tags are aligned with the 
center of antennas. In Fig. 9, the distance $\beta$ from the tag to the center of antenna is larger than $\alpha$. If we suppose that they are on the same plane, it will be inevitable to increase positioning error. In order to obtain the accurate trajectory, we can extend our method to 3D scenario. In this case, the surveillance plane is composed of cubes with mm-level width. However, the computation of tracking tags in 3D space is greatly huger than that in 2D scenario, which will reduce real-time performance. So we will take further study in our future work.

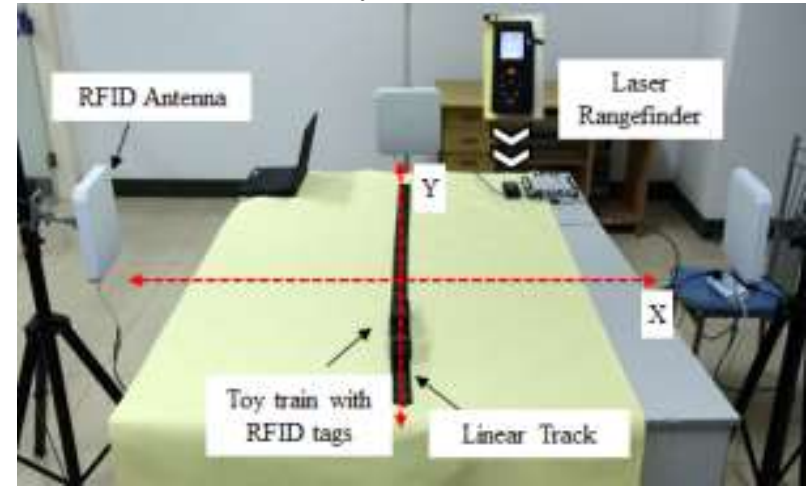

Fig. 10 (a). Linear Track

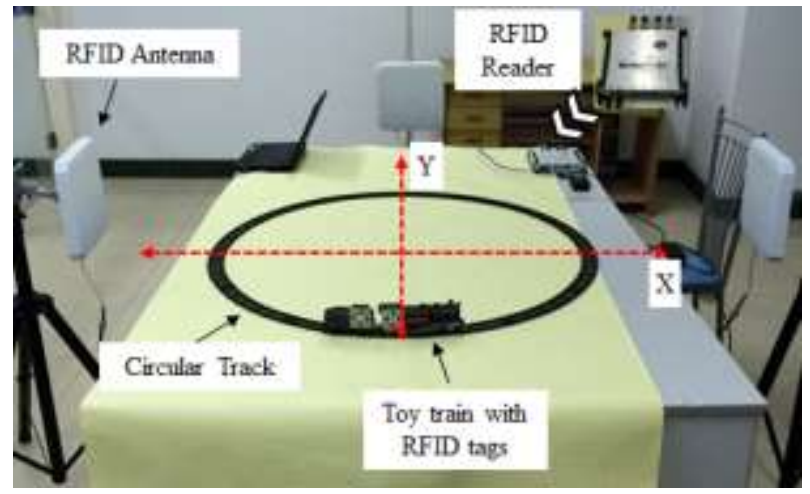

Fig. 10(b). Circular Track

\section{IMPLEMENTATION AND EVALUATION}

In this section, we present the implementation and conduct performance evaluation on the prototype.

\section{A. Implementation}

1) Hardware

We implement our method using an Impinj R420 RFID reader, four 8dBi directional antennas and Impinj H47 RFID tags. The RFID reader is configured as the fastest RF mode (Max Throughput, Dual target and Session 0) to improve read rate. The antennas with the size of $280 \mathrm{~mm} \times 280 \mathrm{~mm} \times 40 \mathrm{~mm}$ are connected to the Impinj R420 reader. In China, UHF RFID operating frequency band is $920.625 \mathrm{MHz} \sim 924.375 \mathrm{MHz}$ with $500 \mathrm{kHz}$ channel spacing. Here, the fixed frequency, 920.625 $\mathrm{MHz}$, is selected in our experiments. A plastic toy train attached with Impinj $\mathrm{H} 47$ tags moves with the constant speed of $20 \mathrm{~cm} / \mathrm{s}$ on the track.

Two kinds of motion trajectories are deployed in our experiment- Linear Track and Circular Track.

Linear Track: Antennas 1,2 and 3,4 are respectively deployed on both sides of the track. In the rectangular coordinate system, the coordinates of the antennas are $(0,-60)$, $(100,-60),(100,50)$ and $(0,50)$ respectively, as shown in Fig. 10 (a). The center of antennas perpendicular to the track is assigned with tags attached on the toy train. The Euclidean distances from antennas 1,2 and 3,4 to the track are respectively $60 \mathrm{~cm}$ and $50 \mathrm{~cm}$. The length of linear track is $80 \mathrm{~cm}$. Then we start the toy train with the speed of $20 \mathrm{~cm} / \mathrm{s}$. Ignoring the non-uniform motion state at the beginning, the initial position of the first tag is $(40,0)$.

Circular Track: Antennas 1,2 and 3,4 are respectively deployed around the surveillance region and the coordinates of the antennas are $(-150,0),(0,-150),(150,0)$ and $(0,150)$ respectively, as shown in Fig. 10 (b). The center of antennas is also assigned with tags. The radius of the circular track is $50 \mathrm{~cm}$. The initial position of the tag is $(50,0)$.

In the following experiment, the grid width of the surveillance region is set to $0.25 \mathrm{~cm}$. All of experiments are implemented in a relatively open indoor environment to reduce the influence of multipath propagation and environmental interference as much as possible.

2) Software

We depend on LLRP Toolkit programmers guide [19] to write RFID applications communicating with the R420 RFID reader in C\#. The software is shown in Fig. 11. The collected RF phase estimates are handled in Matlab R2010a to evaluate the TrackT performance. We run the software at a Lenovo Thinkpad X220i computer equipped with dual processor CPU of Core i3 2370M processors at $2.4 \mathrm{GHz}$.

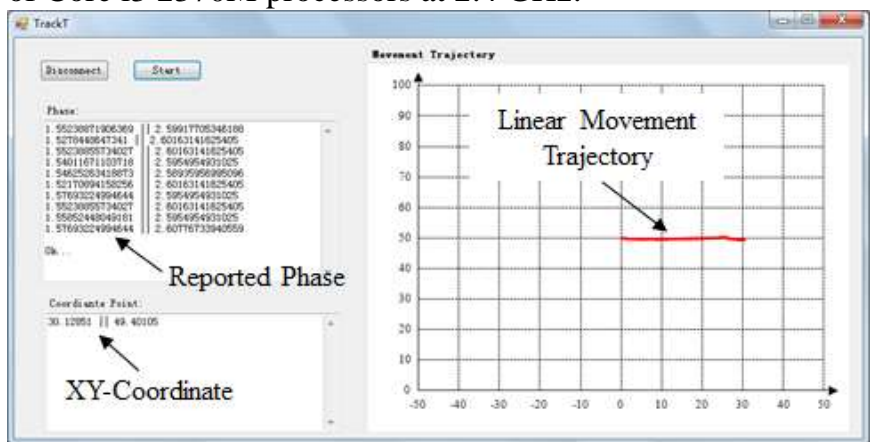

Fig. 11. Software Interface.

\section{B. Evaluation for known track}

1) Error distance between TrackT and DAH

We use the scenario of linear track to evaluate the error distance between TrackT and DAH. The error distance is defined as the Euclidean distance between the ground truth and measured position. In the experiment, the mean of time between the two successive inventories of the same tag for an RFID antenna is about $0.033 \mathrm{~s}$. Since the tag moves along the track at the speed of $20 \mathrm{~cm} / \mathrm{s}$, the mean displacement is about $0.66 \mathrm{~cm}$, far less than $\lambda / 4$. We collect the reported phase from four antennas within 2.5 seconds. The experiment repeats for 50 times respectively. The cumulative distribution function (CDF) of position error based on TrackT and DAH are described in Fig. 12 and 13. TrackT has a mean error distance of $0.18 \mathrm{~cm}, 0.16 \mathrm{~cm}$ and $0.26 \mathrm{~cm}$ in $\mathrm{x}$-axis, $\mathrm{y}$-axis and combined dimension, 
outperforming the DAH method. Most of combined error distances of TrackT are less than $0.5 \mathrm{~cm}$. So TrackT can significantly improve the accuracy of RFID positioning.

2) Impact of tag number

In the experiment, we attach 3-10 tags respectively on the toy

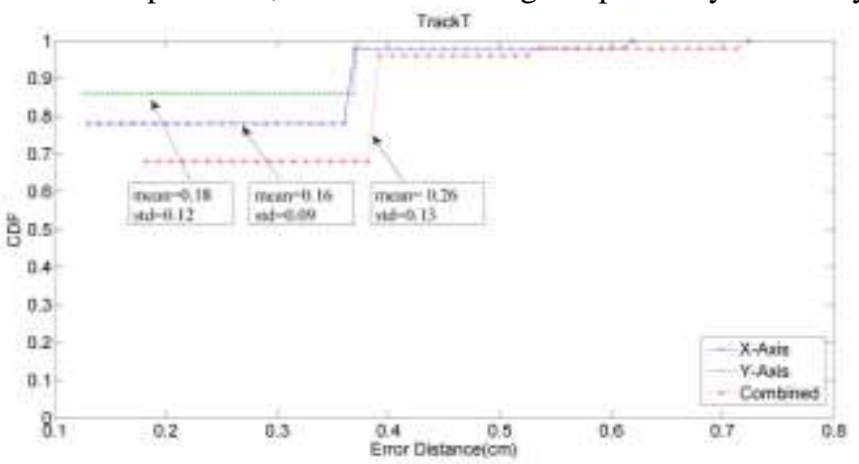

Fig. 12. Tracking in linear motion of RFID tags using TrackT.

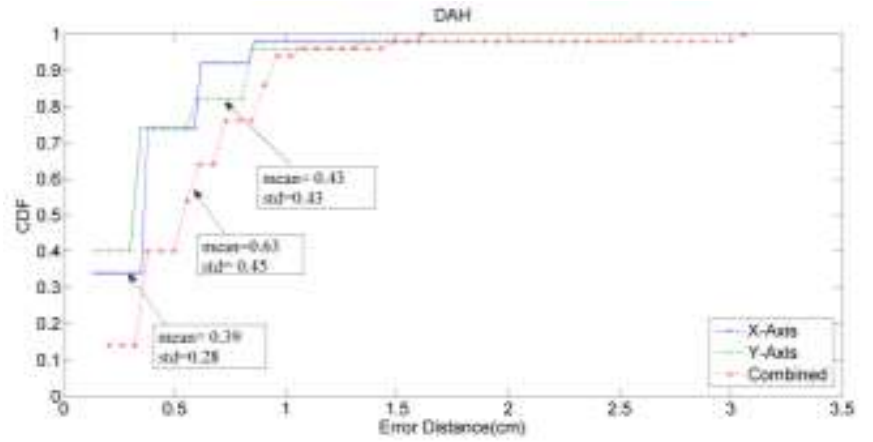

Fig. 13. Tracking in linear motion of RFID tags using DAH.

train to evaluate the impact of tag number. The time to collect phase data is $2.5 \mathrm{~s}$. As the number of RFID tags increases, the mean of time between two successive inventories of the same tag for an RFID antenna will increase accordingly. In this case, the total number of captured phase data for each tag will decrease within the interval of $2.5 \mathrm{~s}$. From Fig. 14, when there are 3 tags attached on the toy train, the mean of combined error distance is $0.38 \mathrm{~cm}$ with the standard deviation of $0.15 \mathrm{~cm}$. However, when there are 10 tags on the toy train, TrackT obtains a mean combined error distance of $0.72 \mathrm{~cm}$ with the standard deviation of $0.31 \mathrm{~cm}$. Therefore, as the number of tags in the surveillance region increases, TrackT should take more time to receive enough reads for keeping the high positioning accuracy.

3) Realtime performance

TrackT is a real-time tracking system. Once the RFID reader collects a measured phase, TrackT will compute the phase difference between the current phase and the former one. The final tracking result will be released after enough reads have been received in the system. As depicted in Fig.15, the accuracy tends towards stability when the total number of reads from all of antennas is more than 70 times. In addition, if there is only one tag in the surveillance region, the time between successive inventories of the same tag is $33 \mathrm{~ms}$ as described before. In this case, the mean of time interval for each calculation should be less than $33 \mathrm{~ms}$ in order to maintain the realtime performance of TrackT. In Section IV, we have discussed how to reduce the computations, so the method is conducted here to evaluate its effectiveness. The experiment repeats for 50 times respectively with optimization and without optimization. Suppose that the computation time of outputting tracking result after 70 reads is $\mathrm{t}$, so the mean computation time between the two successive inventories is $t / 69$.

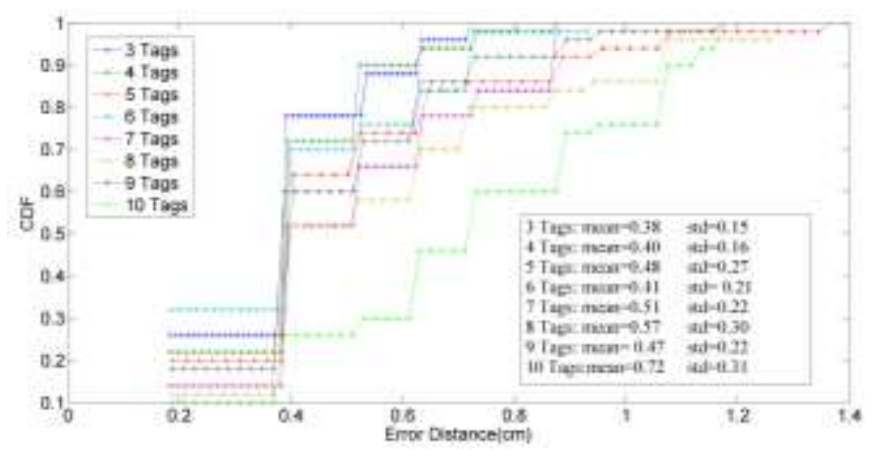

Fig. 14. Impact of tag number. As the number of tracked tags increases, the positioning accuracy will be reduced.

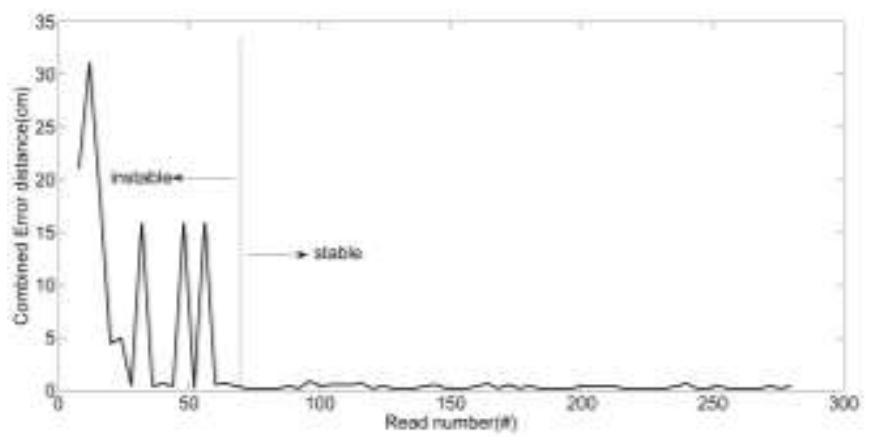

Fig. 15. Stability for known track. The accuracy tends towards stability when the sum of reads from all of antennas is more than 70 times.

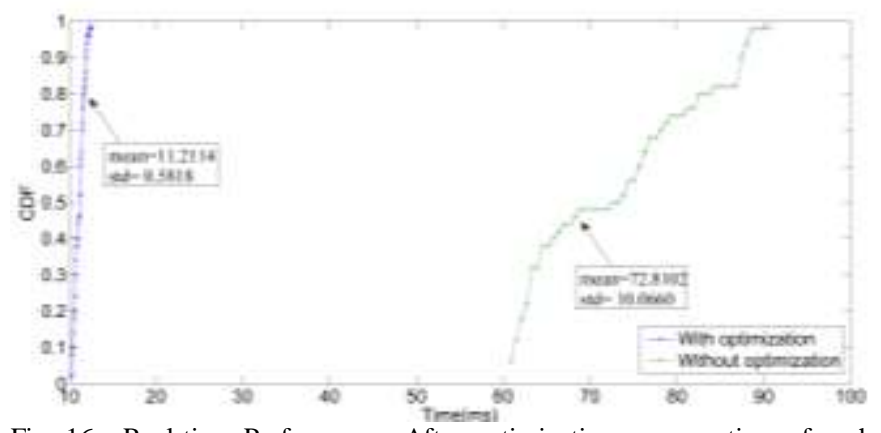

Fig. 16. Real-time Performance. After optimization, a mean time of each calculation is about $11.2114 \mathrm{~ms}$ with a standard deviation $0.5818 \mathrm{~ms}$, which can meet real-time performance.

The experimental results are shown in Fig.16. A mean time of each calculation is $72.81 \mathrm{~ms}$ and a standard deviation is $10.06 \mathrm{~ms}$ without optimization, which far exceeds than $33 \mathrm{~ms}$. The generation with optimization keeps a low time cost, achieving a mean time of $11.21 \mathrm{~ms}$ with a standard deviation $0.58 \mathrm{~ms}$. So the optimization method described in Section IV is effective to make the system meet the real-time requirements.

4) Impact of antenna position

At first, we deploy antennas 1-4 on both sides of the circular track with the initial coordinates of $(0,-150)$, $(100,-150)$, $(100,150)$ and $(0,150)$. As the vertical distance of each antenna to the center of the track varies from $1.5 \mathrm{~m}$ to $6 \mathrm{~m}$, the impact of antenna position on tracking accuracy is shown in Fig.17. With 
the increase of the distance to the track center, the positioning combined error will also increase, and the error mainly comes from the $y$-axis rather than $\mathrm{x}$-axis. However, when we deploy antennas around the circular track and repeat the above experiment, we find that the combined error is unrelated to

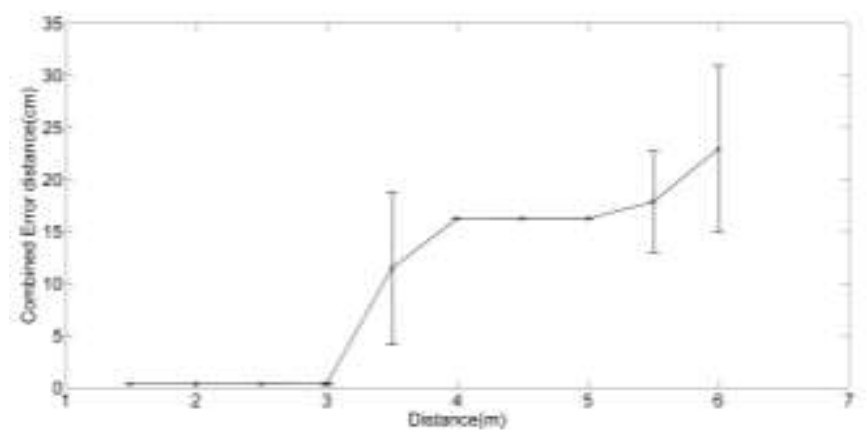

Fig. 17. Impact of antenna position. While antennas are deployed on both sides of the track, the localization error will increase as antennas move away from the track.

distance and the positioning results is absolutely stable with the localization error of about $0.35 \mathrm{~cm}$. From above experiments, the tracking accuracy is related to different deployments of antennas. In the following, we use an example to further discuss such a difference.

Four RFID antennas are deployed on both sides of the circular track, where the coordinates of antennas 1-4 are $(0,-600),(100,-600),(100,600)$ and $(0,600)$ respectively. The initial point of the tracked tag is $(50,0)$. Fig. 18 shows the result of the position correlation for each grid after optimization. The maximum of the position correlation on the grid $\mathrm{W}=100, \mathrm{~L}=65$ is Num $_{100,65}=1346$, so the corresponding coordinate is (50, 32.5 ) and deviates from the ground truth by $32.25 \mathrm{~cm}$. The error mainly comes from the y-axis. Also, there exist many possible candidates because the values of $N u m_{W, \mathrm{~L}}$ on these grids are very close to each other. However, if we deploy antennas around the monitoring area, where the coordinates of antennas $1-4$ are $(-600,0),(0,-600),(600,0)$ and $(0,600)$ respectively, can the error from $\mathrm{x}$-axis and $\mathrm{y}$-axis be cancelled out each other? The maximum on the grid $\mathrm{W}=100, \mathrm{~L}=1$ is $\mathrm{Num}_{100,1}=1174$ in Fig. 19, so the corresponding coordinate is $(50,0.5)$ and deviates from the ground truth by $0.5 \mathrm{~cm}$. Therefore, we can obtain a high accuracy when antennas are deployed around the surveillance region.

From the experiment, when we deploy antennas on the both sides of the track, the distance between antennas and the track should not be far, and it is reasonable to keep it within the $3 \mathrm{~m}$, otherwise there will be a large positioning error. The main reason of decline in accuracy of localization is resulted from dilution of precision (DOP) [31]. Like GPS, the accuracy of RFID tracking system depends on the geometry of reader antennas.

The RFID antennas 1 and 3 in Fig. 19 lie in a direction orthogonal to that of antennas 2 and 4 , giving a relatively small region in which the object tag must lie with some degree of certainty. And the object tag's $\mathrm{X}$ and $\mathrm{Y}$ coordinates are determined with equal precision. For example, in Fig. 20 the uncertainty region (the intersection of the circular lines) is small (low DOP). In Fig. 18, however, the antennas 1, 2 or 3, 4 are very close to each other, resulting in a considerably large uncertainty region, so the positioning accuracy will be diluted in some extent.

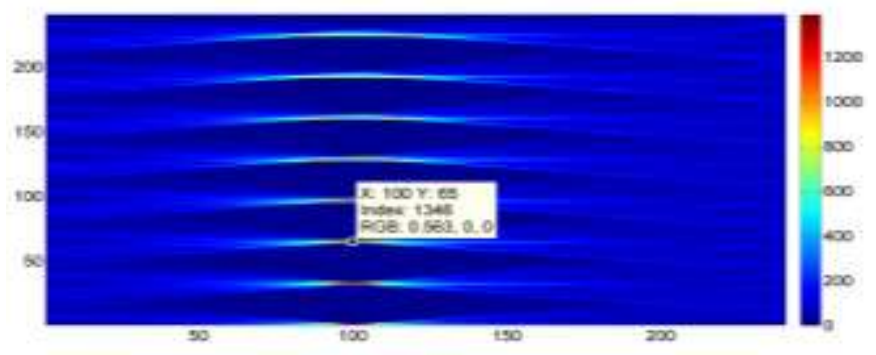

Fig. 18. Position ambiguity while antennas are deployed on both sides of the monitoring area.

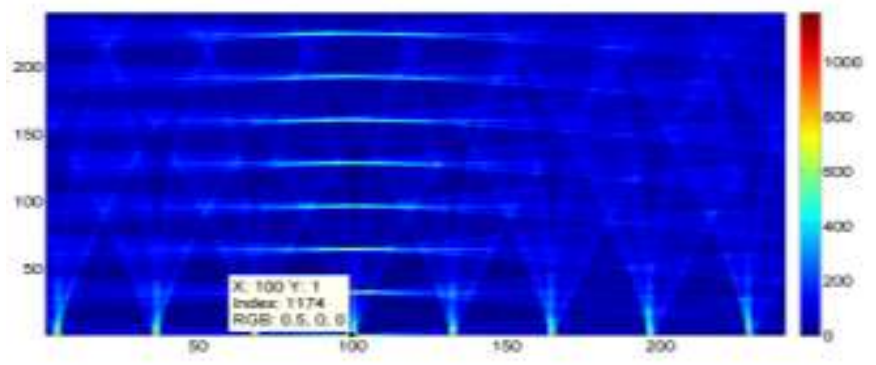

Fig. 19. The elimination of position ambiguity while antennas are deployed around the monitoring area.

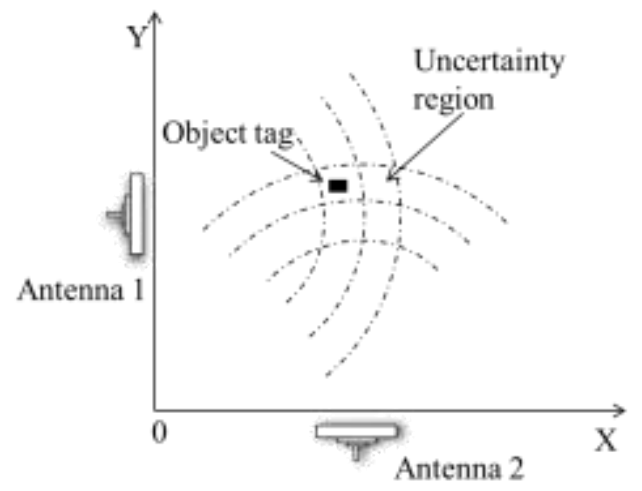

Fig. 20. Low dilution of precision. The RFID antenna 1 lies in a direction orthogonal to that of antenna 2, giving a relatively small region (the intersection of the circular lines), so the position uncertainty of the object RFID tag is small.

As a result, it is a feasible way to reduce the uncertainty region by increasing RFID antennas deployed in the surveillance region. Further, the antennas should be deployed around the track $[32,33]$ to obtain more stable and higher positioning results under the permission condition.

\section{Evaluation for unknown track}

We use the scenario of circular track described before to evaluate our method to track the unknown trajectory. The toy train attached on a tag moves anticlockwise along the track at a constant speed of $20 \mathrm{~cm} / \mathrm{s}$ and the initial position of tag is $(50,0)$. The tag on the toy train moves 10 laps anticlockwise within about 157 seconds. We collect phase data during the time to estimate the tag's moving speed and tracking accuracy. 
1) The estimated speed

At first, we evaluate the speed of tag movement. In Fig. 21, the arrow represents the velocity vector calculated in every read round. In the experiment, the estimated speed has a mean of $19.11 \mathrm{~cm} / \mathrm{s}$ while the ground truth speed is $20 \mathrm{~cm} / \mathrm{s}$. Fig. 22 illustrates that the estimated speed tends towards stability after more than about 200 reads are collected by four antennas.

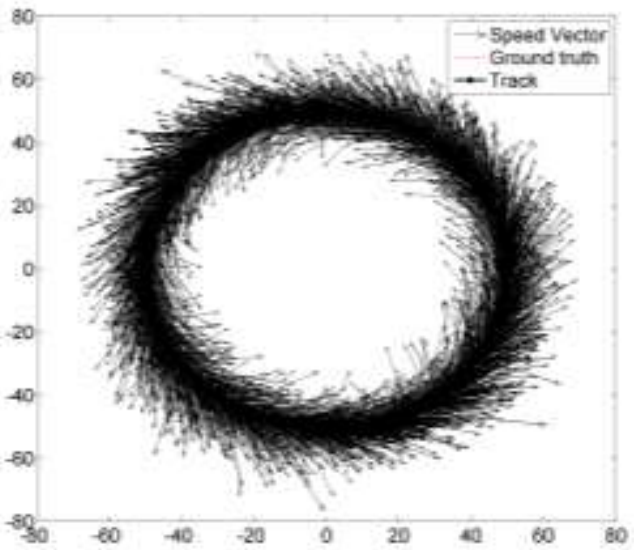

Fig. 21. Estimated speed for unknown track. The estimated speed has a mean of $19.11 \mathrm{~cm} / \mathrm{s}$ while the ground truth speed is $20 \mathrm{~cm} / \mathrm{s}$.

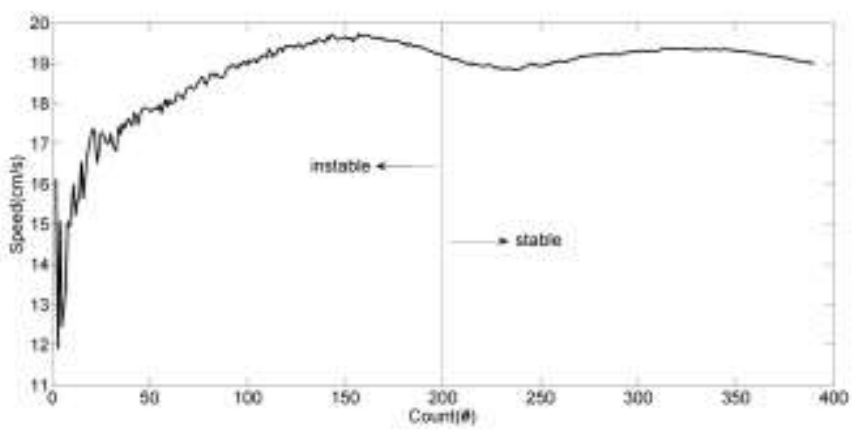

Fig. 22. Stability for unknown track. The estimated speed tends towards stability after more than 200 reads are collected by four antennas.

2) The accuracy between the fixed trajectory and the ground truth

In Fig. 23, each point represents the estimated tag position based on the collected phase in every round while the red line represents the ground truth. In the experiment, we extract the coordinate points whose $\mathrm{x}$-axis coordinates are within $[-50,50]$, to calculate corresponding $\mathrm{y}$-axis coordinates in the circular track, which is regarded as the ground truth in y-axis dimension. So TrackT has a mean error distance of $0.37 \mathrm{~cm}$ with the standard deviation of $0.53 \mathrm{~cm}$ in y-axis dimension. Similarly, we can also acquire a mean error distance of $0.41 \mathrm{~cm}$ with the standard deviation of $0.62 \mathrm{~cm}$ in $\mathrm{x}$-axis dimension by extracting the coordinate points whose $\mathrm{y}$-axis coordinates are within $[-50,50]$. Finally, the mean combined error distance is approximately $0.55 \mathrm{~cm}$ with the standard deviation of $0.75 \mathrm{~cm}$.

\section{Conclusion}

In this paper we mainly achieve to track mobile tags based on phase collected by the COTS RFID reader. The difference of phase periodicity deduced by reported phase has strong tolerance to multipath effect, which is employed to acquire all of candidates in the surveillance area. On this basis, we calculate the double-difference values corresponding to these candidates and select the minimum value as the final position. In addition, since the time between successive inventories of the same tag is short enough and an RFID tag are not moving at

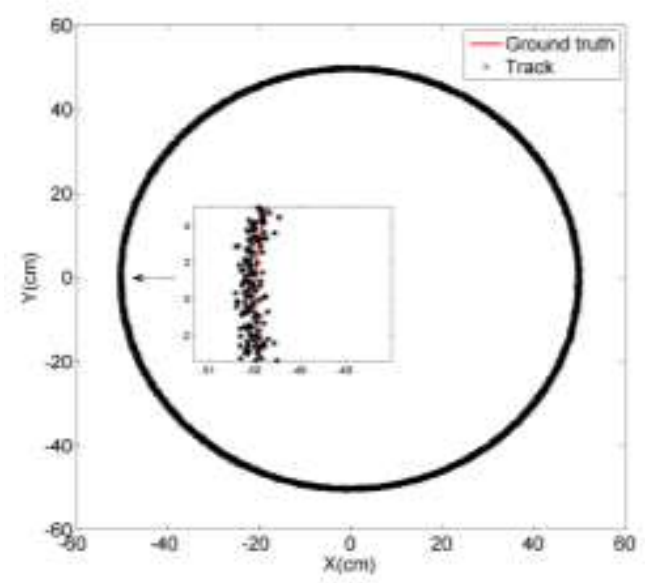

Fig. 23. Tracked positions. Each black point represents the localization result of TrackT in every read round.

a high speed, we use a first-order Taylor series approximation to compute a tag's displacements every round of antenna scheduled. Moreover, the rule of reducing computation is also proposed in this paper to make TrackT meet real-time performance. The results of our experiment demonstrate that TrackT can be applied to track known and unknown movement trajectory with high accuracy in real time.

The limitations and practical deployment issues are as follows:

(i) Tag population size. Our current design is to track a small amount of RFID tags at the same time because the time between successive inventories of the same tag will depend on tag population size. While large-scale RFID tags exist in the surveillance zone, the moving distance will be large than $\lambda / 4$ during the time interval, leading to the failure of our localization system.

(ii) Calibration of antenna coordinates. Before tracking RFID tag movement trajectory, the system needs to calibrate accurate positions of each RFID antenna at first. This is because the phase is very sensitive to the distance between the tag and antenna. We employ a RFID tag fixed on the known position and a laser rangefinder with high measurement accuracy to calibrate the location of RFID antenna, thereby improving localization accuracy.

In the future, we will rely on captured RFID tags' movement trajectory to recognize human motion gestures, which can be used in virtual reality.

\section{REFERENCES}

[1] X. Yang, Y. Zhang and X. Liang, "Primate-inspired communication methods for mobile and static sensors and RFID tags," ACM Transactions on Autonomous and Adaptive Systems (TAAS), vol. 6, no. 4, Article No. 26, Oct. 2011 
[2] X. Yang, S. Yu, K. Wu, Q. Ni, C. Janecek and J. Nordstad, "Radio frequency identification: technologies, applications, and research issues," Wireless Communications and Mobile Computing, vol. 7, no. 4, pp. 457-472, Jul. 2007.

[3] C. Floerkemeier and M. Lampe, "Issues with RFID usage in ubiquitous computing applications," in Proc. Pervasive, 2004, pp. 188-193.

[4] J. Brusey, C. Floerkemeier, M. Harrison and M. Fletcher, "Reasoning about uncertainty in location identification with RFID," in Proc. IJCAI, 2003, pp. 23-30.

[5] L. Yang, Y. Qi, J. Fang, X. Ding, T. Liu and M. Li, "Reasoning about uncertainty in location identification with RFID," in Proc. IEEE INFOCOM, 2014, pp. 2670-2678.

[6] P. Vorst and A. Zell, "Fully autonomous trajectory estimation with long-range passive RFID," in Proc. IEEE ICRA, 2010, pp. 1867-1872.

[7] L.M. Ni, Y. Liu, Y.C. Lau and A.P. Patil, "LANDMARC: indoor location sensing using active RFID," Wireless networks, vol. 10, no. 6, pp. 701-710, Nov. 2004.

[8] C. Wang, H. Wu and N.F. Tzeng, "RFID-based 3-D positioning schemes," in Proc. IEEE INFOCOM, 2007, pp. 1235-1243.

[9] A. Bekkali, H. Sanson and M. Matsumoto, "RFID indoor positioning based on probabilistic RFID map and Kalman filtering," in Proc. IEEE WiMOB, 2007, pp. 21-21.

[10] L. Shangguan, Z. Li, Z. Yang, M. Li and Y. Liu, "Otrack: Order tracking for luggage in mobile RFID systems," in Proc. IEEE INFOCOM, 2013, pp. 3066-3074.

[11] J.D. Griffin and G.D. Durgin, "Complete link budgets for backscatter-radio and RFID systems," IEEE Antennas and Propagation Magazine, vol. 51, no. 2, pp. 11-25, Apr. 2009.

[12] ImpinJ (2013, Sep.). Speedway revolution reader application note: Low level user data support. ImpinJ Inc. Seattle, USA. [Online]. Available: https://support.impinj.com/hc/en-us/articles/202755318-Application-Not e-Low-Level-User-Data-Support

[13] Y. Zhao, Y. Liu and L.M. Ni, "Vire: Active rfid-based localization using virtual reference elimination," in Proc. IEEE ICPP, 2007, pp.56.

[14] C. Hekimian-Williams, B. Grant, X. Liu, Z. Zhang and P. Kumar, "Accurate localization of RFID tags using phase difference," in Proc. IEEE RFID, 2010, pp. 89-96.

[15] S. Azzouzi, M. Cremer, U. Dettmar, R. Kronberger and T. Knie, "New measurement results for the localization of UHF RFID transponders using an Angle of Arrival (AoA) approach," in Proc. IEEE RFID, 2011, pp. 91-97.

[16] P.V. Nikitin, R. Martinez, S. Ramamurthy, H. Leland ,G. Spiess and K. V. S. Rao, "Phase based spatial identification of UHF RFID tags," in Proc. IEEE RFID, 2010, pp.102-109.

[17] T. Liu, L. Yang, Q. Lin, Y. Guo and Y. Liu, "Anchor-free backscatter positioning for RFID tags with high accuracy," in Proc. IEEE INFOCOM, 2014, pp. 379-387.

[18] J. Wang and D. Katabi, "Dude, where's my card?: RFID positioning that works with multipath and non-line of sight," in Proc. ACM SIGCOMM, 2013, pp. 51-62.

[19] L. Yang, Y. Chen, X. Li, C. Xiao, M. Li and Y. Liu, "Tagoram: Real-time tracking of mobile RFID tags to high precision using COTS devices," in Proc. ACM MobiCom, 2014, pp. 237-248.

[20] D.M. Dobkin, The RF in RFID: UHF RFID in Practice, Lincoln, USA: Newnes, 2012, pp. 7-49.

[21] ImpinJ (2015, Jun.). Octane LLRP Guide version 5.4.0. ImpinJ Inc. Seattle, USA. [Online]. Available: https://support.impinj.com/hc/en-us/articles/202755298-Reader-Docume ntation

[22] P. Zhang, J. Gummeson and D. Ganesan, "Blink: A high throughput link layer for backscatter communication," in Proc. ACM MobiSys, 2012, pp. 99-112.

[23] C. Wang, L. Xie and W. Wang, "Moving tag detection via physical layer analysis for large-scale RFID systems," in Proc. IEEE INFOCOM, 2016, pp. 1-9.

[24] H. Mustafa, X. Zhu, Q. Li and G. Chen, "Efficient median estimation for large-scale sensor RFID systems," International Journal of Sensor Networks, vol. 12, no. 3, pp. 171-183, Nov. 2012.

[25] G. Ferrari, F. Cappelletti and R. Raheli, "A simple performance analysis of RFID networks with binary tree collision arbitration," International Journal of Sensor Networks, vol. 4,no.3, pp. 194 - 208, 2008.

[26] B. Hofmann-Wellenhof, H. Lichtenegger and J. Collins, "Global positioning system: theory and practice," New York, USA: Springer Science \& Business Media, 2013, pp. 258-264.
[27] A.F.C. Errington, B.L.F. Daku and A.F. Prugger, "Initial position estimation using RFID tags: A least-squares approach," IEEE Trans. Instrumentation and Measurement, vol. 59, no. 11, pp. 2863-2869, Nov. 2010.

[28] B. Gu, V. S. Sheng, K. Y. Tay, W. Romano, and S. Li. 2015. Incremental support vector learning for ordinal regression. Wireless networks, vol. 26, no. 7, pp. 1403-1416, July 2015.

[29] S. Sen, J. Lee, K. H. Kim and P. Congdon, "Avoiding multipath to revive inbuilding WiFi localization," in Proc. ACM MobiSys, 2013, pp. 249-262.

[30] J. Xiong and K. Jamieson, "ArrayTrack: A Fine-Grained Indoor Location System," in Proc. NSDI, 2013, pp. 71-84.

[31] R. B. Langley, "Dilution of precision," GPS world, vol. 10, no. 5, pp. 52-59, May 1999.

[32] Z. Wang, N. Ye, R. Malekian, R. Wang, Peng Li,"TMicroscope: Behavior Perception Based on the Slightest RFID Tag Motion", Elektronika ir Elektrotechnika, Vol.22, No.2, pp.114-122,2016.

[33] N. Ye, Z. Q. Wang, R. Malekian, Q. Lin, R. Wang, "A Method for Driving Route Predictions Based on Hidden Markov Model", Mathematical Problems in Engineering, Vol.2015, pp.1-12, 2015.

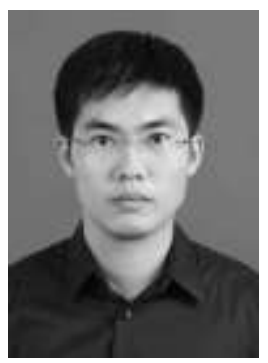

Zhongqin Wang (STM'16) received the Master degree in Computer Software and Theory from Nanjing University of Posts and Telecommunications, Nanjing, China in 2014. He is currently pursuing the Ph.D. degree at Nanjing University of Posts and Telecommunications. In 2016, he became a Student Member of IEEE. His research interest includes Radio Frequency Identification (RFID), sensor network and fancy Human Interaction.

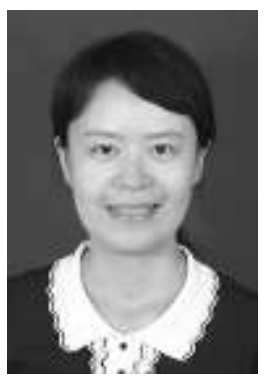

Ning Ye received the B.S. degree in Computer Science from Nanjing University in 1994, the M.S. degree in School of Computer \& Engineering from Southeast University in 2004, and the Ph.D. degree in Institute of Computer Science from Nanjing University of Posts and Telecommunications in 2009. She is currently a Professor there. In 2010, Ning Ye worked as a Visiting Scholar and Research Assistant in the Department of Computer Science, University of Victoria, Canada. Her research interests include information processing in wireless networks and Internet of Things. She is a senior member of Chinese Computer Federation (CCF).

Reza Malekian received the B.Eng. degree in computer engineering, the M.Eng. degree in telecommunications engineering, and the Ph.D. degree in computer science. He is currently a Senior Lecturer with the

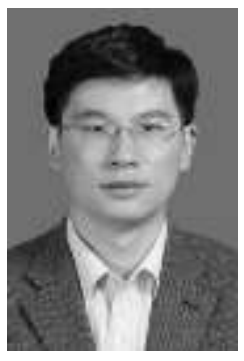

Department of Electrical, Electronic and Computer Engineering, University of Pretoria, South Africa. His research lies in the area of advanced sensor networks, Internet of Things, and mobile communications. He is also a Professional Member of the British Computer Society and Chartered Engineer.

Fu Xiao (M'12) received the Ph.D Degree in Computer Science and Technology from Nanjing University of Science and Technology, Nanjing, China, in 2007. He is 
currently a Professor and $\mathrm{PhD}$ supervisor with the School of Computer, Nanjing University of Posts and Telecommunications, Nanjing, China. His main research interest is Wireless Sensor Networks. Dr. Xiao is a member of the IEEE Computer Society and the Association for Computing Machinery.

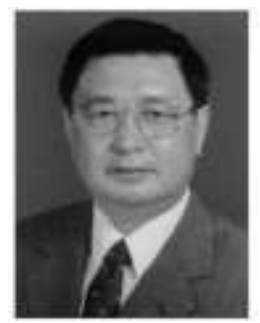

Ruchuan Wang was born in Anhui Province, China. He researched on graphic processing at University of Bremen and program design theory Ludwig Maximilian Muenchen Unitversitaet from 1984 to 1992 . He is a professor and tutor of Ph.D. candidate in Nanjing University of Posts and Telecommunications since 1992. Major research interests include wireless sensor networks and information security. 